\title{
The moduli space of embedded singly periodic maximal surfaces with isolated singularities in the Lorentz-Minkowski space $\mathbb{L}^{3}$
}

\author{
Isabel Fernández * Francisco J. López * Rabah Souam *
}

\begin{abstract}
We show that, up to some natural normalizations, the moduli space of singly periodic complete embedded maximal surfaces in the Lorentz-Minkowski space $\mathbb{L}^{3}=$ $\left(\mathbb{R}^{3}, d x_{1}^{2}+d x_{2}^{2}-d x_{3}^{2}\right)$, with fundamental piece having a finite number $(n+1)$ of singularities, is a real analytic manifold of dimension $3 n+4$. The underlying topology agrees with the topology of uniform convergence of graphs on compact subsets of $\left\{x_{3}=0\right\}$.
\end{abstract}

\section{Introduction}

A maximal surface in the Lorentz-Minkowski space $\mathbb{L}^{3}=\left(\mathbb{R}^{3}, d x_{1}^{2}+d x_{2}^{2}-d x_{3}^{2}\right)$ is a spacelike surface with zero mean curvature. It locally maximizes the area functional associated to variations by spacelike surfaces. In a pioneering work, Calabi 2] proved that the affine spacelike planes are the only complete maximal surfaces in $\mathbb{L}^{3}$ (Calabi, in fact, showed the analogous result for maximal hypersurfaces in $\mathbb{L}^{4}$ and this was later extended to maximal hypersurfaces in $\mathbb{L}^{n}$, for all $n$, by Cheng and Yau [3]). Nonetheless, recent works show there is a rich global theory of complete maximal surfaces with singularities in $\mathbb{L}^{3}$ ([8], 7], 10, 13, 14). For instance, Umehara and Yamada 14 have obtained results on the global behavior of immersed maximal surfaces having analytic curves of singularities. Of particular interest are the complete embedded maximal surfaces having a closed discrete set of singularities. First it should be remarked that maximal surfaces in $\mathbb{L}^{3}$ share some properties with minimal surfaces in the Euclidean space $\mathbb{R}^{3}$. Indeed, Kobayashi [12] gave a Weierstrass type representation in terms of meromorphic data similar to the one of minimal surfaces in $\mathbb{R}^{3}$. Also, both types of surfaces are locally represented as graphs of solutions of

*Research partially supported by MCYT-FEDER grant number MTM2004-00160. 2000 Mathematics Subject Classification. Primary 53C50; Secondary 58D10, 53C42.

Key words and phrases: maximal surfaces, periodic surfaces, conelike singularities 


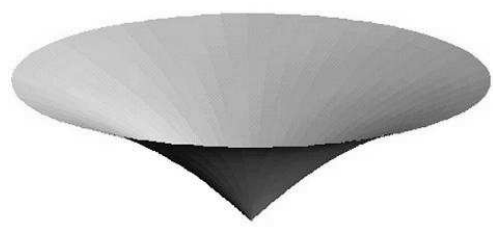

Figure 1: The Lorentzian catenoid

elliptic operators. An important difference, however, is that the maximal surface equation for graphs in $\mathbb{L}^{3}$ may have solutions with isolated singularities and this never happens for the minimal surface equation in $\mathbb{R}^{3}$. Otherwise said, an embedded maximal surface can have an isolated singularity, contrarily to an embedded minimal surface in $\mathbb{R}^{3}$. This is illustrated by the Lorentzian half-catenoid, [12] (see Figure 1). At a singular point around which a maximal surface is embedded in $\mathbb{L}^{3}$, the Gauss curvature blows up, the limit tangent planes become lightlike and the surface is asymptotic to a half lightcone at the singularity (cf. [10, [12 and [8]). For these reasons, such points are called conelike singularities.

Embedded complete maximal surfaces with a closed discrete set of singularities are global graphs over any spacelike plane. Conformally, the regular set of such a surface is a Riemann surface minus as many pairwise disjoint closed conformal disks (without accumulation points) as singular points in the surface. A fundamental observation is that the Weierstrass data for the surface extends to the double Riemann surface, [7. This allows one to work on boundaryless Riemann surfaces.

In [8] we developed the theory of embedded complete maximal surfaces in $\mathbb{L}^{3}$ of finite type, that is those having a finite number of singularities. We showed, in particular, that the moduli space of such surfaces with $n+1 \geq 2$ singularities and vertical limit normal at infinity is a $3 n+4$-dimensional manifold.

The next simplest subclass of maximal surfaces in $\mathbb{L}^{3}$ with a closed discrete set of singularities consists of the periodic ones with finite type in the quotient. This refers to surfaces that are invariant under a discrete group $G$ of isometries of $\mathbb{L}^{3}$ acting freely and properly and such that the quotient surfaces are embedded and have a finite number of singularities in the flat and complete 3-dimensional Lorentzian manifold $\mathbb{L}^{3} / G$. In [7], the first and second author proved fundamental facts about the global geometry of these surfaces. In particular, they classified the discrete groups $G \subset I s o\left(\mathbb{L}^{3}\right)$ for which $\mathbb{L}^{3} / G$ contains complete maximal surfaces of finite type. If $\mathbb{L}^{3} / G$ is orientable and orthochronous (i.e the elements of $G$ preserve the orientation and the future time direction) and contain a complete embedded maximal surface $S$ of finite type then $G$ is a group of spacelike translations of rank one or two and $S$ is an annulus of finite conformal type or a torus, respectively.

In this paper, we study the moduli space of (embedded) singly periodic maximal surfaces in $\mathbb{L}^{3}$ having finite type in the quotient space. That is, we consider the group $\langle T>$ generated by a spacelike translation $T$ in $\mathbb{L}^{3}$ and complete embedded maximal surfaces with a finite number of singularities in $\mathbb{L}^{3} /\langle T\rangle$. Several examples of this kind were 
constructed in [7] (see figure 21).

First note that up to an ambient isometry of $\mathbb{L}^{3}$ and rescaling, we can suppose that $T=(1,0,0)$. In this case any complete embedded maximal surface with a finite number of singularities in $\mathbb{L}^{3} /\langle(1,0,0)\rangle$ is a graph over the cylinder $\left\{x_{3}=0\right\} \subset \mathbb{L}^{3} /\langle(1,0,0)\rangle$. We also normalize so that one of the ends of the surface is asymtpotic to $\left\{x_{3}=0, x_{2} \geq 0\right\}$. Our main result then says that:

The space $\mathfrak{M}_{n}$ of marked entire maximal graphs over the cylinder $\left\{x_{3}=0\right\}$ in $\mathbb{L}^{3} /<(1,0,0)>$ having $n+1 \geq 2$ conelike singularities (the mark is an ordering of the set of singularities) and an end asymptotic to $\left\{x_{3}=0, x_{2} \geq 0\right\}$, is a real analytic manifold of dimension $3 n+4$. A global coordinate system is given by the ordered sequence of points in the mark and the normal to the second end. This space is a $(n+1)$ !-sheeted covering of the space $\mathfrak{G}_{n}$ of (non marked) entire maximal graphs over the cylinder $\left\{x_{3}=0\right\}$ in $\mathbb{L}^{3} /\langle(1,0,0)\rangle$, having $n+1 \geq 2$ conelike singularities and an end asymptotic to $\left\{x_{3}=0, x_{2} \geq 0\right\}$. The underlying topology of $\mathfrak{G}_{n}$ is equivalent to the uniform convergence of graphs on compact subsets of the cylinder $\left\{x_{3}=0\right\}$.

We have organized our paper as follows: Section 2 contains some preliminaries about the local behavior of maximal surfaces around isolated singularities and the global behavior of complete maximal surfaces of finite type in the quotient space $\mathbb{L}^{3} /\langle T\rangle$. Section 3 is devoted to the proof of the main theorem. Our approach relies on algebraic geometry tools: we define some natural bundles on the moduli space $\mathcal{T}_{n}$ of once punctured marked circular domains with $n+1$ boundary components (a mark is an ordering of the boundary circles), and introduce a spinorial bundle $\mathcal{S}_{n}$ associated to the moduli space of Weierstrass data of surfaces in the space of graphs with $n+1$ singularities. The convergence in $\mathfrak{M}_{n}$ means convergence of marked conformal structures in $\mathcal{T}_{n}$ and of Weierstrass data.

\section{Preliminaries}

We denote by $\overline{\mathbb{C}}, \mathbb{D}$ the extended complex plane $\mathbb{C} \cup\{\infty\}$ and the unit disc $\{z \in \mathbb{C}:|z|<$ $1\}$, respectively.

Throughout this paper, $\mathbb{L}^{3}$ will denote the three dimensional Lorentz-Minkowski space $\left(\mathbb{R}^{3},\langle\rangle,\right)$, where $\langle\rangle=,d x_{1}^{2}+d x_{2}^{2}-d x_{3}^{3}$. By definition, a coordinate system $\left(y_{1}, y_{2}, y_{3}\right)$ in $\mathbb{L}^{3}$ is said to be a $(2,1)$-coordinate system if the Lorentzian metric is given by $d y_{1}^{2}+d y_{2}^{2}-d y_{3}^{3}$. We say that a vector $\mathbf{u} \in \mathbb{R}^{3}-\{\mathbf{0}\}$ is spacelike, timelike or lightlike if $\|u\|^{2}:=\langle\mathbf{u}, \mathbf{u}\rangle$ is positive, negative or zero, respectively. When $u$ is spacelike, $\|u\|$ is chosen non negative. The vector $\mathbf{0}$ is spacelike by definition. A plane in $\mathbb{L}^{3}$ is spacelike, timelike or lightlike if the induced metric is Riemannian, non degenerate and indefinite or degenerate, respectively.

We call $\mathbb{H}^{2}=\left\{\left(x_{1}, x_{2}, x_{3}\right) \in \mathbb{R}^{3}: x_{1}^{2}+x_{2}^{2}-x_{3}^{2}=-1\right\}$ the hyperbolic sphere in $\mathbb{L}^{3}$ of constant intrinsic curvature -1 . Note that $\mathbb{H}^{2}$ has two connected components $\mathbb{H}_{+}^{2}:=$ $\mathbb{H}^{2} \cap\left\{x_{3} \geq 1\right\}$ and $\mathbb{H}_{-}^{2}:=\mathbb{H}^{2} \cap\left\{x_{3} \leq-1\right\}$. The stereographic projection $\sigma$ for $\mathbb{H}^{2}$ is defined 
as follows:

$$
\sigma: \overline{\mathbb{C}}-\{|z|=1\} \longrightarrow \mathbb{H}^{2} ; z \rightarrow\left(\frac{2 \operatorname{Im}(z)}{|z|^{2}-1}, \frac{-2 \operatorname{Re}(z)}{|z|^{2}-1}, \frac{|z|^{2}+1}{|z|^{2}-1}\right),
$$

where $\sigma(\infty)=(0,0,1)$.

By definition, an isometry in $\mathbb{L}^{3}$ is said to be orthochronous if its associated linear isometry preserves $\mathbb{H}_{+}^{2}$ (and so $\mathbb{H}_{-}^{2}$ ). In other words, it preserves the future direction.

In the sequel, $\mathcal{N}$ will denote a complete flat 3-dimensional Lorentzian manifold (i.e., a 3-dimensional differential manifold endowed with a flat metric of index one). It is well known that the universal isometric covering of $\mathcal{N}$ is $\mathbb{L}^{3}$ (see for example [16], 15]). Thus $\mathcal{N}$ can be regarded as the quotient of $\mathbb{L}^{3}$ under the action of a discrete group $G$ of isometries acting freely and properly on $\mathbb{L}^{3}$.

In what follows, $\mathcal{M}$ will denote a differentiable surface.

An immersion $X: \mathcal{M} \longrightarrow \mathcal{N}$ is spacelike if the tangent plane at any point is spacelike, that is to say, the induced metric on $\mathcal{M}$ is Riemannian. In this case, $S=X(\mathcal{M})$ is said to be a spacelike surface in $\mathcal{N}$. If $\mathcal{N}=\mathbb{L}^{3} / G$, where $G$ is a (possibly trivial) group of translations acting freely and properly on $\mathbb{L}^{3}$, the locally well defined Gauss map $N_{0}$ of $X$ assigns to each point of $\mathcal{M}$ a point of $\mathbb{H}^{2}$. A connectedness argument gives that $N_{0}$ is globally well defined and $N_{0}(\mathcal{M})$ lies, up to a Lorentzian isometry, in $\mathbb{H}_{-}^{2}$. This means that $\mathcal{M}$ is orientable.

A maximal immersion $X: \mathcal{M} \longrightarrow \mathcal{N}$ is a spacelike immersion with null mean curvature. In this case, $S=X(\mathcal{M})$ is said to be a maximal surface in $\mathcal{N}$. Using isothermal parameters, $\mathcal{M}$ can be endowed with a conformal structure. In the orientable case, $\mathcal{M}$ becomes a Riemann surface.

Theorem 2.1 (Weierstrass representation of maximal surfaces in $\mathbb{L}^{3}[12]$ ) Let $X$ : $\mathcal{M} \rightarrow \mathbb{L}^{3}$ be a conformal maximal immersion of a Riemann surface. Then $g \stackrel{\text { def }}{=} \sigma^{-1} \circ N_{0}$ is a meromorphic function, and there exists a holomorphic 1-form $\phi_{3}$ defined on $\mathcal{M}$ such that

(i) the 1-forms given by $\phi_{1}=\frac{i}{2}\left(\frac{1}{g}-g\right) \phi_{3}$ and $\phi_{2}=\frac{-1}{2}\left(\frac{1}{g}+g\right) \phi_{3}$ are holomorphic on $\mathcal{M}$

(ii) the induced Riemannian metric on $M$ is given by $d s^{2}=\left|\phi_{1}\right|^{2}+\left|\phi_{2}\right|^{2}-\left|\phi_{3}\right|^{2}=\frac{1}{4}\left(\frac{1}{|g|}-\right.$ $|g|)^{2}\left|\phi_{3}\right|^{2}$

(iii) for any closed curve $\gamma$ in $M$ we have $\operatorname{Re} \int_{\gamma}\left(\phi_{1}, \phi_{2}, \phi_{3}\right)=0$

(iv) up to a translation, the immersion is given by $X=R e \int_{P_{0}}\left(\phi_{1}, \phi_{2}, \phi_{3}\right)$, where $P_{0} \in M$ is an arbitrary point.

Conversely, given $g$ and $\phi_{3}$ a meromorphic function and a holomorphic 1-form on $M$, respectively, such that $(i),($ ii $)$ and $($ iii $)$ are satisfied, then $(i v)$ defines a conformal maximal immersion of $M$ in $\mathbb{L}^{3}$. 
Remark 2.1 (Weierstrass data of maximal surfaces in translational spaces) The Weierstrass data $\left(\phi_{1}, \phi_{2}, \phi_{3}\right)$ of a maximal surface in $\mathbb{L}^{3}$ are invariant by translations. Therefore, maximal surfaces in a quotient $\mathbb{L}^{3} / G$, where $G$ is a group of translations acting properly and freely, also have a Weierstrass representation as above except that the condition (iii) is replaced by the following one:

$\left(i i i^{\prime}\right)$ for any closed curve $\gamma$ in $M$ the translation of associated vector $\operatorname{Re} \int_{\gamma}\left(\phi_{1}, \phi_{2}, \phi_{3}\right)$ is an element of the group $G$.

Definition 2.1 Let $X: \mathcal{M} \rightarrow \mathcal{N}$ be a topological embedding of a smooth surface in a Lorentzian 3-manifold and $F \subset \mathcal{M}$ a closed discrete subset. We say that $X$ is a maximal embedding with singular set $F$ if $\left.X\right|_{\mathcal{M}-F}$ is a maximal embedding and the induced metric on $\mathcal{M}-F$ converges to zero at any point of $F$.

In this case we say that $S=X(\mathcal{M})$ is a maximal surface in $\mathcal{N}$ with singularities at $X(F)$.

Lemma 2.1 (structure of embedded singularities in $\mathbb{L}^{3}[8]$, [4]) Let $X: \mathcal{D} \rightarrow \mathbb{L}^{3}$ be a maximal embedding defined on an open disk $\mathcal{D}$, with an isolated singularity at $q \in \mathcal{D}$.

Then $\mathcal{D}-\{q\}$ is conformally equivalent to $\{z \in \mathbb{C}: r<|z|<1\}$, for some $r>0$, and if $X_{0}:\{r<|z|<1\} \rightarrow \mathbb{L}^{3}$ is a conformal reparameterization of $X$, then $X_{0}$ extends analytically to $A_{r}:=\{1 \leq|z|<1\}$ by setting $X_{0}(\{|z|=1\})=P_{0}:=X(q)$.

The Weierstrass data $\left(g, \phi_{3}\right)$ of $X_{0}$ satisfy: $g$ is injective and $|g|=1$ on $\{|z|=1\}$, and $\phi_{3}(z) \neq 0,|z|=1$. In particular, $X_{0}$ reflects analytically about $\{|z|=1\}$ to the mirror surface $A_{r}^{*}:=\{z \in \mathbb{C}: 1 \leq|z|<1 / r\}$, verifying $g \circ J=1 / \bar{g}$ and $J^{*}\left(\phi_{3}\right)=-\bar{\phi}_{3}$, where $J(z)=1 / \bar{z}$ is the mirror involution.

Moreover for any spacelike plane $\Pi$ plane containing $P_{0}$ the Lorentzian orthogonal projection $\pi: X(\mathcal{D}) \rightarrow \Pi$ is a local homeomorphism and $X(\mathcal{D})$ is asymptotic near $P_{0}$ to a half light cone with vertex at $P_{0}$.

The point $P_{0}$ is said to be a conelike singularity of $X(\mathcal{D})$.

Remark 2.2 The universal covering of a complete flat Lorentzian 3-dimensional manifold is isometric to $\mathbb{L}^{3}$ (cf. [15]). Therefore the above Lemma extends to the more general context of complete flat Lorentzian 3-manifolds.

As a consequence of the previous lemma, if $X: \mathcal{M} \rightarrow \mathcal{N}$ is a complete embedded maximal surface with a closed discrete set $F \subset \mathcal{M}$ of singularities, where $\mathcal{N}$ is complete and flat, then $\mathcal{M}-F$ is conformally equivalent to $\Sigma-\cup_{p \in F} D_{p}$, where $\Sigma$ is a Riemann surface and the $\left\{D_{p}\right\}_{p \in F}$ are closed pairwise disjoint conformal disks with no accumulation in $\Sigma$.

We also have that the conformal reparameterization $X: \Sigma-\cup_{p \in F} D_{p} \rightarrow \mathcal{N}$ extends analytically to $\mathcal{M}_{0}:=\Sigma-\cup_{p \in F} \operatorname{Int}\left(D_{p}\right)$, by putting $X\left(\partial D_{p}\right)=X(p)$ for each $p \in F$. In the sequel we will refer to $\mathcal{M}_{0}$ as the conformal support of the embedding $X$. We also say that $\mathcal{M}_{0}$ is the conformal support of the maximal surface $X(\mathcal{M}) \subset \mathcal{N}$.

In particular, if $\mathcal{N}=\mathbb{L}^{3}$ or $\mathcal{N}=\mathbb{L}^{3} / G$, where $G$ is a translational group, the Weierstrass data $\left(\phi_{1}, \phi_{2}, \phi_{3}\right)$ extend analytically to $\mathcal{M}_{0}$. 
We denote by $\mathcal{M}_{0}^{*}$ the mirror surface of $\mathcal{M}_{0}$ and by $N$ the double surface, that is, $N=\mathcal{M}_{0} \cup \mathcal{M}_{0}^{*}$ with the identification $\partial\left(\mathcal{M}_{0}\right) \equiv \partial\left(\mathcal{M}_{0}^{*}\right)$. Moreover, we call $J: N \rightarrow N$ the antiholomorphic mirror involution, and observe that the fixed point set of $J$ coincides with $\partial\left(\mathcal{M}_{0}\right)$. By Lemma 2.1, the Weierstrass data $\Phi:=\left(\phi_{1}, \phi_{2}, \phi_{3}\right)$ can be extended by Schwartz reflection to $N$ satisfying $J^{*}(\Phi)=-\bar{\Phi}$.

For the sake of simplicity, complete maximal embedded surfaces with a finite set of singularities in $\mathcal{N}$ will be called CMF surfaces.

Definition 2.2 (flux of a closed curve) Let $S \subset \mathcal{N}$ be an oriented $C M F$ surface, and let $X: \mathcal{M}_{0} \rightarrow \mathcal{N}$ be a conformal reparameterization of $S$.

For any closed curve $\gamma(s)$ in $\mathcal{M}_{0}$ parameterized by the arclength, we label $\nu$ as its unit conormal vector so that $\left\{\nu, \gamma^{\prime}\right\}$ is positive with respect to the orientation in $\mathcal{M}_{0}$. The flux vector of the curve $\gamma$ is defined as

$$
F(\gamma):=\int_{\gamma} \nu(s) d s
$$

Since $X$ is harmonic it follows from Stokes theorem that $F(\gamma)$ depends only on the homology class of $\gamma$ in $\mathcal{M}_{0}$. If $\mathcal{N}=\mathbb{L}^{3}$ or $\mathbb{L}^{3} / G$, where $G$ is translational, and we denote by $\Phi=\left(\phi_{1}, \phi_{2}, \phi_{3}\right)$ the Weierstrass data of $X$, it is easy to check that

$$
F(\gamma)=\operatorname{Im}\left(\int_{\gamma} \Phi\right)
$$

We define the flux at a conelike singularity $q \in S$ as the flux along any curve homotopic to the boundary component $\gamma_{0}$ of $\mathcal{M}_{0}$ corresponding to $q$. It can be checked hat the flux at conelike singularity is always a timelike vector (see [11]).

Definition 2.3 (CMSF surfaces) A complete embedded maximal surface $\tilde{S} \subset \mathbb{L}^{3}$ with a closed discrete set of singularities is said to be singly periodic of finite type if:

- $\tilde{S}$ is invariant under the free and proper action of an infinite cyclic group $G$ of isometries of $\mathbb{L}^{3}$

- $\tilde{S} / G$ is a $C M F$ surface in $\mathbb{L}^{3} / G$.

In the sequel $\tilde{S}$ will be called for short a CMSF surface.

Remark 2.3 There is a natural connection between CMSF surfaces in $\mathbb{L}^{3}$ and CMF surfaces in quotients $\mathbb{L}^{3} / G$, where $G$ is cyclic.

As stated in the above definition a CMSF surface determines a CMF surface in the corresponding space $\mathbb{L}^{3} / G$. Conversely, Theorem [2.2 below will show that the universal covering of a CMF surface in $\mathbb{L}^{3} / G$ is a CMSF surface in $\mathbb{L}^{3}$. 


\subsection{Representation of CMSF surfaces}

In the sequel we will denote by $\langle u\rangle$ the cyclic group generated by the translation of vector $u \in \mathbb{L}^{3}$.

Theorem $2.2([7])$ Let $S$ be a CMF surface with $n+1$ singular points in $\mathbb{L}^{3} / G$, where $G$ is a cyclic group of isometries of $\mathbb{L}^{3}$ acting properly and freely. Then

- The CMSF surface $\tilde{S}$ obtained by lifting $S$ to $\mathbb{L}^{3}$ is an entire graph over any spacelike plane.

- The group $G$ is generated by a spacelike translation $T$.

- The conformal support $\mathcal{M}_{0}$ of $S$ is $\mathbb{C}^{*}-\cup_{i=0}^{n} \operatorname{Int}\left(D_{i}\right)$ where the $D_{i}, i=0, \ldots, n$ are pairwise disjoint closed (circular) disks. The associated double surface $N$ is $\bar{N}$ $\{0, \infty, J(0), J(\infty)\}$, where $\bar{N}$ is a compact Riemann surface of genus $n$, the points $0, \infty \in \bar{N}$ correspond to the ends of $\mathcal{M}_{0}$ and $J$ denotes the mirror involution.

- If $X: \mathcal{M}_{0} \rightarrow \mathbb{L}^{3} / G$ is a conformal parameterization of $S, X$ applies each boundary circle $\partial\left(D_{i}\right)$ to a singular point of $S$.

- The Weierstrass data $\Phi:=\left(\phi_{1}, \phi_{2}, \phi_{3}\right)$ of $X$ can be extended by Schwarz reflection to $N$, satisfying: $J^{*}(\Phi)=-\bar{\Phi}$.

Moreover $\Phi$ has simple poles at $0, \infty, J(0)$ and $J(\infty)$, and the topological ends of $S$ are of Scherk type, that is to say, asymptotic to spacelike flat half cylinders in $\mathbb{L}^{3} / G$.

- $T$ can be chosen as the translation of vector $u=\operatorname{Re} \int_{\gamma} \Phi$, where $\gamma \subset \mathcal{M}_{0}$ is a closed loop around 0.

Conversely given $\mathcal{M}_{0}:=\mathbb{C}^{*}-\cup_{i=0}^{n} \operatorname{Int}\left(D_{i}\right)$ where the $D_{i}, i=0, \ldots, n$ are pairwise disjoint closed (circular) disks, define $N$ and $J$ as before and take a Weierstrass data $\Phi$ on $N$ satisfying $J^{*}(\Phi)=-\bar{\Phi}$ and having simple poles at the ends. Then

$$
X: \mathcal{M}_{0} \rightarrow \mathbb{L}^{3} /\langle u\rangle \quad X=\operatorname{Re} \int_{P_{0}} \Phi
$$

where $u=\operatorname{Re} \int_{\gamma} \Phi$, defines a complete embedded maximal surface with $n+1$ singularities and its universal covering is a CMSF surface in $\mathbb{L}^{3}$ invariant under the group $\langle u\rangle$.

\subsection{Uniqueness of CMSF surfaces}

Theorem 2.3 (Uniqueness) Let $S_{i}$ denote a CMF surface in $\mathbb{L}^{3} /\langle u\rangle$ with singular points $q_{1}^{i}, \ldots, q_{n}^{i} \in \mathbb{L}^{3} /\langle u\rangle, i=1,2$ where $u \in\left\{x_{3}=0\right\}, u \neq 0$. Suppose $S_{1}$ and $S_{2}$ are contained in $\left\{x_{3} \geq 0\right\} \subset \mathbb{L}^{3} /\langle u\rangle$, have the same limit normal directions at the ends and that $q_{j}^{1}=q_{j}^{2}$, $j=1, \ldots, n$. Then $S_{1}=S_{2}$. 

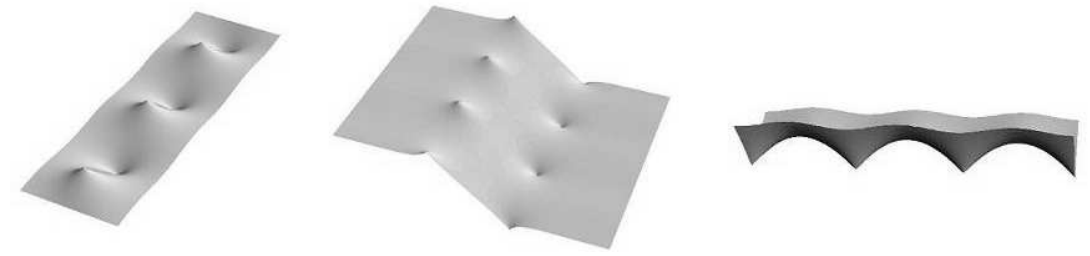

Figure 2: Examples of CMSF surfaces

Proof: The proof is based on the maximum principle. A regular maximal surface in $\mathbb{L}^{3}$ can be represented locally as a graph $x_{3}=u\left(x_{1}, x_{2}\right)$ of a smooth function $u$, with $u_{x_{1}}^{2}+u_{x_{2}}^{2}<1$, satisfying the equation:

$$
\left(1-u_{x_{1}}^{2}\right) u_{x_{2} x_{2}}+2 u_{x_{1}} u_{x_{2}} u_{x_{1} x_{2}}+\left(1-u_{x_{2}}^{2}\right) u_{x_{1} x_{1}}=0 .
$$

The maximum principle for elliptic quasilinear equations then gives the following geometric maximum principles for maximal surfaces:

Let $\tilde{S}_{1}$ and $\tilde{S}_{2}$ be two maximal embedded surfaces (possibly with boundary) in $\mathbb{L}^{3}$ which intersect tangentially at a point $p$. Suppose that locally, around $p, \tilde{S}_{1}$ is above $\tilde{S}_{2}$, that is to say, $u_{1} \geq u_{2}$ where $u_{i}$ denotes the function defining the graph $\widetilde{S}_{i}, i=1,2$. Then $\tilde{S}_{1}=\tilde{S}_{2}$ locally around $p$ if one of the following hypotheses holds:

- $p$ is an interior point of $\tilde{S}_{1}$ and $\tilde{S}_{2}$,

- $p$ is a boundary point of $\tilde{S}_{1}$ and $\tilde{S}_{2}$ and $\partial \tilde{S}_{1}$ and $\partial \tilde{S}_{2}$ are tangent at $p$.

In either case, by analyticity of solutions of elliptic equations, we also infer that the two graphs $\tilde{S}_{1}$ and $\tilde{S}_{2}$ coincide whenever they are simultaneously defined. It is important to emphasize that this local statement only works for maximal graphs without singularities.

Consider now $S_{1}$ and $S_{2}$ as in the statement of the theorem. Since $u$ is a horizontal vector, vertical translations are well defined isometries of $\mathbb{L}^{3} /\langle u\rangle$. For any $t \in \mathbb{R}$, put $S_{i}(t)=S_{i}+(0,0, t), i=1,2$.

¿From our assumptions and the asymptotic behavior of the ends given in Theorem 2.2 , we deduce that, for $t>0$ big enough, $S_{1}(t)>S_{2}$, that is to say $S_{1}(t) \cap S_{2}=\emptyset$ and $S_{1}(t)$ is above $S_{2}$. Let $t_{0}=\inf \left\{t>0: S_{1}(t)>S_{2}\right\}$. We are going to prove that $t_{0}=0$.

Suppose $t_{0}>0$. If $S_{1}\left(t_{0}\right) \cap S_{2} \neq \emptyset$ then $S_{1}\left(t_{0}\right)$ and $S_{2}$ have a contact point different from the singularities. But then the interior maximum principle implies that $S_{1}\left(t_{0}\right)=S_{2}$, which is absurd.

Assume now that $S_{1}\left(t_{0}\right) \cap S_{2}=\emptyset$ (contact at infinity). Because the ends of the surfaces are asymptotic to spacelike flat half cylinders, then for $\epsilon>0$ small enough, $S_{1}\left(t_{0}-\epsilon\right) \cap S_{2}$ is a non empty compact real 1-dimensional analytic manifold containing a Jordan curve $\Gamma$ spanning two parallel annular ends without singular points $E_{1} \subset S_{1}\left(t_{0}-\epsilon\right)$ and $E_{2} \subset S_{2}$, with $E_{1} \cap E_{2}=\Gamma$. Let $F_{1}=\int_{\Gamma} \nu_{1}$ and $F_{2}=\int_{\Gamma} \nu_{2}$ the flux along $\Gamma$ in $S_{1}\left(t_{0}-\epsilon\right)$ and $S_{2}$ resp. (see definition 2.2). It is not hard to see that $F_{i}$ is orthogonal to $u$ and to the limit normal 
vector at the end $E_{i}$. Moreover, $\left\langle F_{i}, F_{i}\right\rangle=\langle u, u\rangle$, and so we infer that $F_{1}=F_{2}$. However by the boundary maximum principle, the third coordinate of $\nu_{1}$ is strictly bigger than that of $\nu_{2}$ along $\Gamma$, which is a contradiction. This proves that $t_{0}=0$, and reversing the argument, that $S_{1}=S_{2}$.

Corollary 2.1 The group of ambient isometries preserving a CMF surface $S$ in $\mathbb{L}^{3} /\langle u\rangle$ coincides with:

- the group of orthochronous (i.e., preserving the future direction) ambient isometries leaving invariant the set of its singularities and preserving the set of normal directions at the ends in case the ends of $S$ are not parallel

- the group of ambient isometries leaving invariant the set of its singularities and the limit normal vector at the ends in case $S$ has parallel ends.

\section{The space of CMSF surfaces}

In this section we are going to study the moduli space of CMSF surfaces in $\mathbb{L}^{3}$. By Remark 2.3. this space can be identified with the space of CMF surfaces in quotients $\mathbb{L}^{3} / G$, where $G=\langle u\rangle$ and $u$ is a spacelike vector. Moreover, as shown in Theorem 2.2, we can restrict ourselves to the case of CMF graphs over spacelike flat cylinders in $\mathbb{L}^{3} /\langle u\rangle$.

First, we have to introduce some normalizations.

Let $\tilde{S}$ be a CMSF surface invariant by a spacelike translation $T$. Up to a isometry in $\mathbb{L}^{3}$ and rescaling we will always suppose $T(p)=p+(1,0,0)$. From Theorem 2.2 we know that $S:=\tilde{S} /\langle(1,0,0)\rangle$ is a CMF graph over the cylinder $\left\{x_{3}=0\right\} \subset \mathbb{L}^{3} /\langle(1,0,0)\rangle$ with flat ends. Up to a hyperbolic rotation in $\mathbb{L}^{3} /\langle(1,0,0)\rangle$ we can suppose that one of them is asymptotic to the half cylinder $\left\{x_{3}=0, x_{2} \geq 0\right\}$.

In the sequel we denote by $\mathfrak{G}_{n}$ the space of CMF graphs in $\mathbb{L}^{3} /\langle(1,0,0)\rangle$ over the cylinder $\left\{x_{3}=0\right\}$ with one of their ends, which will be denoted $E_{1}$ in the sequel, asymptotic to $\left\{x_{3}=0, x_{2} \geq 0\right\}$ and having $n+1$ singularities, $n \geq 1$. We will always suppose that all $S \in \mathfrak{G}_{n}$ are oriented by the past directed normal. Note that the limit normal to the second end $E_{2}$ of $S$ lies in $\mathbb{H}^{2} \cap\left\{x_{3}<0, x_{1}=0\right\}$. The latter set is identified, through a suitable stereographic projection, with the real interval $]-1,+1[$.

Let $S \in \mathfrak{G}_{n}$ and label $F$ as its set of singularities. By definition, a mark in $S$ is an ordering $\mathfrak{m}=\left(q_{0}, q_{1}, \ldots, q_{n}\right) \in\left(\mathbb{L}^{3} /\langle(1,0,0)\rangle\right)^{n+1}$ of the points in $F$, and we say that $(S, \mathfrak{m})$ is a marked graph. We denote by $\mathfrak{M}_{n}$ the space of marked graphs and define the two following maps:

$$
\begin{array}{ccc}
\mathfrak{s}_{1}: \mathfrak{M}_{n} \rightarrow \mathfrak{G}_{n} & \text { and } & \left.\mathfrak{s}_{2}: \mathfrak{M}_{n} \rightarrow\left(\mathbb{L}^{3} /\langle(1,0,0)\rangle\right)^{n+1} \times\right]-1,1[ \\
\mathfrak{s}_{1}(S, \mathfrak{m})=S & \mathfrak{s}_{2}(S, \mathfrak{m})=(\mathfrak{m}, c)
\end{array}
$$


where $c \in]-1,+1\left[\right.$ is the limit normal at the end $E_{2}$ as explained above.

Label $\mathcal{P}_{n+1}$ as the symmetric group of permutations of order $n+1$ and denote by $\mu: \mathcal{P}_{n+1} \times \mathfrak{M}_{n} \rightarrow \mathfrak{M}_{n}$, the natural action $\mu(\tau,(S, \mathfrak{m})):=(S, \tau(\mathfrak{m}))$. Observe that the space $\mathfrak{G}_{n}$ can be naturally identified with the orbit space of this action.

This section is devoted to prove the main result of this paper:

Main Theorem The set $\left.\mathfrak{s}_{2}\left(\mathfrak{M}_{n}\right) \subset\left(\mathbb{L}^{3} /\langle(1,0,0)\rangle\right)^{n+1} \times\right]-1,+1[$ is open and the one to one map $\mathfrak{s}_{2}: \mathfrak{M}_{n} \rightarrow \mathfrak{s}_{2}\left(\mathfrak{M}_{n}\right)$ provides a global system of analytic coordinates on $\mathfrak{M}_{n}$.

Moreover, the action $\mu$ is discontinuous and hence $\mathfrak{G}_{n}$ has a unique analytic structure making $\mathfrak{s}_{1}$ an analytic covering of $(n+1)$ ! sheets.

This section is organized as follows: in Subsection 3.1 we identify $\mathfrak{M}_{n}$ with a set $\mathcal{S}_{n} \times$ $\mathbb{L}^{3} /\langle(1,0,0)\rangle \times\{-1,1\}$, where $\mathcal{S}_{n}$ is a divisor bundle associated to the Weierstrass data. The definition of $\mathcal{S}_{n}$ involves some elements of classical theory of Riemann surfaces, like the Jacobian variety and the Abel-Jacobi map, which will be explained in this subsection.

In Subsection 3.2 we prove that $\mathcal{S}_{n}$ has a natural structure of differentiable $(3 n+$ 1)-manifold, and thus we use the previous identification to endow $\mathfrak{M}_{n}$ with a structure of differentiable manifold of dimension $3 n+4$.

Finally in Subsection 3.3 we prove the Main Theorem, first showing that $\mathfrak{s}_{2}$ is smooth when we consider the previous differentiable structure on $\mathfrak{M}_{n}$, and then applying the Domain Invariance Theorem.

\subsection{Identifying $\mathfrak{M}_{n}$}

We split this subsection into three stages.

\subsubsection{From marked graphs in $\mathfrak{M}_{n}$ to divisors on marked circular domains}

The following definition and notations are required.

We label $\mathcal{T}_{n} \subset \mathbb{R}^{3 n+2}$ as the $(3 n+1)$-dimensional connected analytical submanifold consisting of points $v=\left(c_{0}, c_{1}, \ldots, c_{n}, r_{0}, r_{1}, \ldots r_{n}\right)$ in $] 1,+\infty\left[\times \mathbb{C}^{n} \times\left(\mathbb{R}^{+}\right)^{n+1}\right.$ such that $r_{0}=c_{0}-1$, the discs $D_{j}:=\left\{\left|z-c_{j}\right| \leq r_{j}\right\}, j=0,1, \ldots, n$, are pairwise disjoint and $0 \notin D_{j}$, for any $j$. We call $a_{j}:=\partial D_{j}$ and write $c_{j}(v):=c_{j}, r_{j}(v):=r_{j}, D_{j}(v):=D_{j}$ and $a_{j}(v):=a_{j}, j=0,1, \ldots, n$.

Definition 3.1 Given $v \in \mathcal{T}_{n}$, the domain $\Omega(v):=\overline{\mathbb{C}}-\cup_{j=0}^{n} D_{j}(v)$ is said to be a marked circular domain (with $n+1$ holes).

Two marked circular domains $\Omega\left(v_{1}\right)$ and $\Omega\left(v_{2}\right)$ are considered equal if and only if $v_{1}=$ $v_{2}$. 
For any $v \in \mathcal{T}_{n}$, we call $\overline{\Omega(v)}^{*}$ and $N(v)$ the mirror of $\overline{\Omega(v)}:=\Omega(v) \cup\left(\cup_{j=0}^{n} \partial a_{j}(v)\right)$ and the double surface of $\overline{\Omega(v)}$, respectively. Recall that

$$
N(v)=\overline{\Omega(v)} \cup \overline{\Omega(v)^{*}}
$$

with the identification $\partial(\overline{\Omega(v)}) \equiv \partial\left(\overline{\Omega(v)^{*}}\right)$. We know that $N(v)$ is a closed Riemann surface, and $\overline{\Omega(v)^{*}} \cap \overline{\Omega(v)}$ consists of the $n+1$ analytic circles $a_{j}(v), j=0, \ldots, n$. Moreover, we denote by $J_{v}: N(v) \rightarrow N(v)$ the antiholomorphic involution applying any point to its mirror image. Note that the fixed point set of $J_{v}$ coincides with $\cup_{j=0}^{n} a_{j}(v)$.
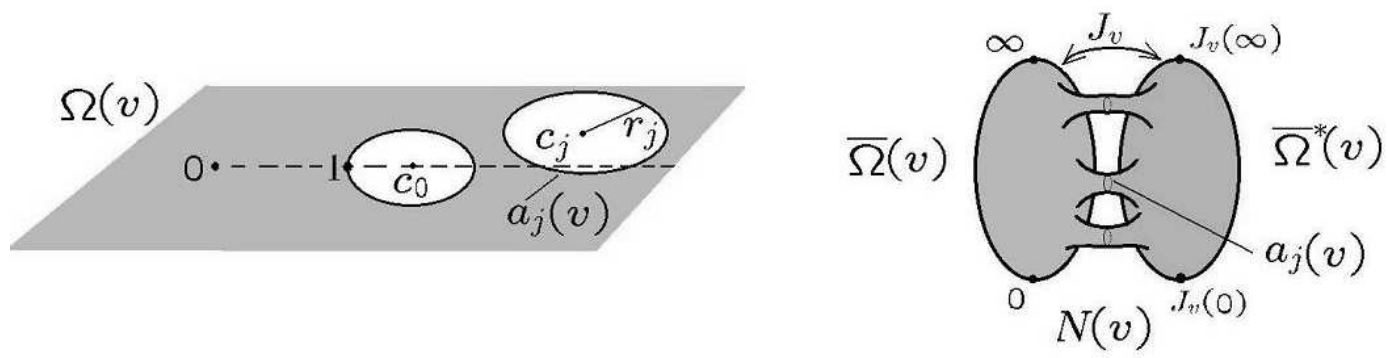

Figure 3: $\Omega(v), N(v)$ and $J_{v}$.

Remark 3.1 A conformal model for $\overline{\Omega(v)}^{*}, v \in \mathcal{T}_{n}$, consists of the planar domain $\overline{\Omega(v)}^{*}:=$ $\left\{J_{v}(z): z \in \overline{\Omega(v)}\right\}$, where

$$
J_{v}(z):=c_{0}(v)+\frac{r_{0}(v)^{2}}{\bar{z}-c_{0}(v)}
$$

is the Schwarz reflection about $a_{0}(v)=\left\{\left|z-c_{0}(v)\right|=r_{0}\right\}$. Moreover, $N(v)$ can be identified to the quotient of $\overline{\Omega(v)} \cup \overline{\Omega(v)}^{*}$ under the identification $z \equiv J_{v}(z), z \in \partial \Omega(v)$.

Let $Y=(S, \mathfrak{m}) \in \mathfrak{M}_{n}$. By Theorem 2.2 we know that the conformal support of $S$ with the prescribed orientation is biholomorphic to a twice punctured circular domain, where the two punctures $\{0, \infty\}$ correspond to the ends and the boundary circles to the singularities (without loss of generality, $E_{1}$ corresponds to $z=0$ ).

We can therefore associate to $(Y, \mathfrak{m})$ a unique element $\Omega(v) \in \mathcal{T}_{n}$ and conformal immersion $X: \overline{\Omega(v)}-\{0, \infty\} \rightarrow \mathbb{L}^{3} /\langle(1,0,0)\rangle$, as stated in the following lemma:

Lemma 3.1 Given $Y=(S, \mathfrak{m}) \in \mathfrak{M}_{n}$, where $\mathfrak{m}=\left(q_{0} \ldots, q_{n}\right)$, there are unique $v \in \mathcal{T}_{n}$ and conformal maximal immersion $X: \overline{\Omega(v)}-\{0, \infty\} \rightarrow \mathbb{L}^{3} /\langle(1,0,0)\rangle$ such that:

(i) $S-F$ is biholomorphic to $\Omega(v)-\{0, \infty\}$ (in the sequel, they will be identified),

(ii) $S=X(\overline{\Omega(v)}-\{0, \infty\})$,

(iii) $z=0$ correspond to the end $E_{1}$, 
(iv) $q_{j}=X\left(a_{j}(v)\right), j=0, \ldots, n$.

Lemma 2.1 and Theorem 2.2 also give that the Weierstrass data of $X,\left(g, \phi_{3}\right)$, satisfy the symmetries $g \circ J_{v}=1 / \bar{g}$ and $J_{v}^{*}\left(\phi_{3}\right)=-\bar{\phi}_{3}$, and that $g$ has exactly $n+1$ zeros $0, w_{1}, \ldots, w_{n} \in \Omega(v)$ counted with multiplicity.

Therefore, putting $D=w_{1} \cdot \ldots \cdot w_{n} \in \operatorname{Div}_{n}(\Omega(v))$, it is easy to see that divisors for the Weierstrass data must be:

$$
(g)=\frac{D \cdot 0}{J_{v}(D \cdot 0)} \quad \text { and } \quad\left(\phi_{3}\right)=\frac{D \cdot J_{v}(D)}{\infty \cdot J_{v}(\infty)}
$$

Since the divisor $D$ determines uniquely the data $\left(g, \phi_{3}\right)$ up to multiplicative constants, and these data control the immersion $X$, we infer that the couple $(v, D)$ encloses all the information about the surface.

\subsubsection{The Abel-Jacobi map on the bundle of divisors}

In order to understand the moduli space $\mathfrak{M}_{n}$, it is crucial to control the structure of the family of couples $(v, D)$ for which there exist Weierstrass data $\left(g, \phi_{3}\right)$ satisfying Equation (11). The Abel-Jacobi map (defined below) will play here a fundamental role.

We need some extra notation.

Given a Riemann surface $\mathcal{R}$, we denote by

$$
\operatorname{Div}_{k}(\mathcal{R})=\{D: D \text { is an integral multiplicative divisor on } \mathcal{R} \text { of degree } k\}
$$

Recall that $\operatorname{Div}_{k}(\mathcal{R})$ is the quotient of $\mathcal{R}^{k}$ under the action of the group of permutations of order $k$, and we denote by $p_{k}: \mathcal{R}^{k} \rightarrow \operatorname{Div}_{k}(\mathcal{R})$ the canonical projection. We endow $\operatorname{Div}_{k}(\mathcal{R})$ with the natural analytic structure induced by $p_{k}$.

In what follows, for any $k \in \mathbb{N}$, we denote by

$$
\mathfrak{D i v}_{k}=\bigcup_{v \in \mathcal{T}_{n}} \operatorname{Div}_{k}(\Omega(v))=\left\{(v, D): v \in \mathcal{T}_{n}, D \in \operatorname{Div}_{k}(\Omega(v))\right\}
$$

and we refer to it as the bundle of $k$-divisors.

Obviously, $\mathfrak{D i v}_{k}$ is a real analytical manifold (see [8] for more details).

Let $\mathcal{J}(v)$ be the Jacobian variety of the compact Riemann surface $N(v)$ associated to the following canonical homology basis:

We identify the homology classes of the boundary circles $a_{j}(v)$ in $\Omega(v)$ with their representing curves $j=0,1, \ldots, n$. Note first that $J_{v}$ fixes $a_{j}(v)$ pointwise, and so, $J_{v}\left(a_{j}(v)\right)=$ $a_{j}(v)$. Take a curve $\gamma_{j} \subset \overline{\Omega(v)}$ joining $a_{0}(v)$ to $a_{j}(v)$, in such a way that the curve $b_{j}(v)$ obtained by joining $\gamma_{j}$ and $J_{v}\left(\gamma_{j}\right)$ satisfies that the intersection numbers $\left(b_{j}(v), b_{h}(v)\right)$ vanish, and $\left(a_{j}(v), b_{h}(v)\right)=\delta_{j h}$, where $\delta_{j h}$ refers to the Kronecker symbol. Observe that $J_{v}\left(b_{j}(v)\right)=-b_{j}(v)$ in the homological sense, and its homology class does not depend on the choice of $\gamma_{j}$. In other words, the identity $J_{v}\left(b_{j}(v)\right)=-b_{j}(v)$ characterizes 


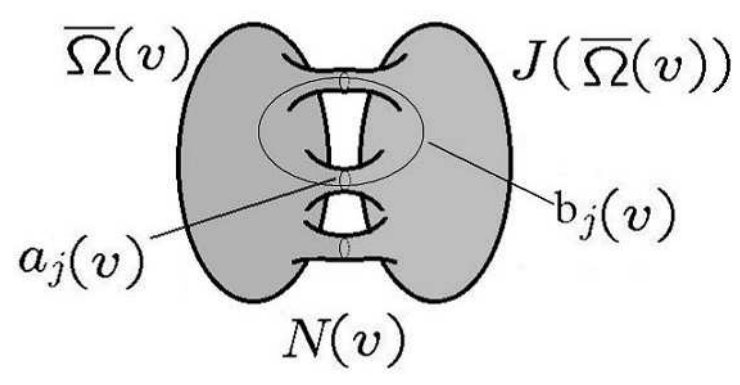

Figure 4: The canonical homology basis $B(v)$

$B(v)=\left\{a_{1}(v), \ldots, a_{n}(v), b_{1}(v), \ldots, b_{n}(v)\right\}$ as canonical homology basis of $N(v)$ (see Figure 4 ).

Call $\left\{\eta_{1}(v), \ldots, \eta_{n}(v)\right\}$ the dual basis of $B(v)$ for the space of holomorphic 1-forms on $N(v)$, that is to say, the unique basis satisfying $\int_{a_{k}(v)} \eta_{j}(v)=\delta_{j k}, j, k=1, \ldots, n$, and put $\Pi(v)=\left(\pi_{j, k}(v)\right)_{j, k=1, \ldots, n}$ for the associated matrix of periods, $\pi_{j, k}(v)=\int_{b_{j}(v)} \eta_{k}(v)$.

Then the Jacobian variety of $N(v)$ is $\mathcal{J}(v)=\mathbb{C}^{n} / L(v)$, where $L(v)$ is the lattice over $\mathbb{Z}$ generated by $\left\{e^{1}, \ldots, e^{n}, \pi^{1}(v), \ldots, \pi^{n}(v)\right\}$, where

$$
e^{j}={ }^{T}\left(0, \ldots,{ }_{1}^{j}, \ldots, 0\right) \text { and } \pi^{j}(v)={ }^{T}\left(\pi_{1, j}(v), \ldots, \pi_{n, j}(v)\right)
$$

The Jacobian bundle is defined as

$$
\mathcal{J}_{n}=\bigcup_{v \in \mathcal{T}_{n}} \mathcal{J}(v)
$$

$\mathcal{J}_{n}$ has a natural structure of analytic manifold (see [8] for more details).

For any $v \in \mathcal{T}_{n}$, we call $\varphi_{v}: N(v) \rightarrow \mathcal{J}(v)$ the Abel-Jacobi embedding defined by

$$
\varphi_{v}(z)=\mathfrak{p}_{v}\left(\int_{1}^{z}{ }^{T}\left(\eta_{1}(v), \ldots, \eta_{n}(v)\right)\right)
$$

where $\mathfrak{p}_{v}: \mathbb{C}^{n} \rightarrow \mathcal{J}(v)$ is the canonical projection (recall that $1 \in \overline{\Omega(v)} \subset N(v)$ uniformly on $v)$. We extend $\varphi_{v}$ with the same name to the Abel-Jacobi map $\varphi_{v}: \operatorname{Div}_{k}(N(v)) \rightarrow \mathcal{J}(v)$ given by

$$
\varphi_{v}\left(P_{1} \cdot \ldots \cdot P_{k}\right)=\sum_{j=1}^{k} \varphi_{v}\left(P_{j}\right), \quad k \geq 1 .
$$

We also define $\varphi: \mathfrak{D i v}_{k} \rightarrow \mathcal{J}_{n}$ by $\varphi(v, D)=\left(v, \varphi_{v}(D)\right)$ 


\subsubsection{The identification $\mathfrak{M}_{n} \equiv \mathcal{S}_{n} \times \mathbb{L}^{3} /\langle(1,0,0)\rangle \times\{-1,1\}$}

Summarizing, we know that given $Y=(S, \mathfrak{m}) \in \mathfrak{M}_{n}$ its associated Weierstrass data (defined on $N(v)$ for the unique $v \in \mathcal{T}_{n}$ given in Lemma 3.1 ) satisfy Equation (11). Abel's Theorem gives

$$
\varphi_{v}(D \cdot 0)-\varphi_{v}\left(J_{v}(D \cdot \infty)\right)=0 \quad \text { and } \quad \varphi_{v}\left(D \cdot J_{v}(D)\right)-\varphi_{v}\left(\infty \cdot J_{v}(\infty)\right)=T(v)
$$

where $T(v) \in \mathcal{J}(v)$ is the image by $\varphi_{v}$ of the divisor associated to a meromorphic 1-form on $N(v)$. By Abel's theorem, $T(v)$ is independent of the choice of the meromorphic 1-form (see []).

These two equations lead to $2 \varphi_{v}(D \cdot 0)-\varphi_{v}\left(0 \cdot \infty \cdot J_{v}(0) \cdot J_{v}(\infty)\right)=T(v)$

Therefore, it is natural to define, for any $v \in \mathcal{T}_{n}$,

$$
\mathcal{S}_{n}(v):=\left\{D \in \operatorname{Div}_{n}(\Omega(v)): 2 \varphi_{v}(D \cdot 0)=T(v)+\varphi_{v}\left(0 \cdot \infty \cdot J_{v}(0) \cdot J_{v}(\infty)\right)\right\},
$$

and also

$$
\mathcal{S}_{n}:=\left\{(v, D): v \in \mathcal{T}_{n}, D \in \mathcal{S}_{n}(v)\right\}
$$

We refer to $\mathcal{S}_{n}$ as the spinorial bundle.

Definition 3.2 With the previous notation, we call $\mathcal{E}$ the map given by

$$
\begin{gathered}
\mathcal{E}: \mathfrak{M}_{n} \rightarrow \mathcal{S}_{n} \times \mathbb{L}^{3} /\langle(1,0,0)\rangle \times\{-1,1\} \\
\mathcal{E}(S, \mathfrak{m})=\left((v, D), q_{0}, \varepsilon_{0}\right)
\end{gathered}
$$

where $\mathfrak{m}=\left(q_{0}, \ldots, q_{n}\right)$ and $\varepsilon_{0} \in\{-1,1\}$ is the sign of the third coordinate of the flux at $q_{0}$ (see Definition 2.2)

The main goal of this paragraph is to show that $\mathcal{E}$ is bijective. Note that the first coordinate of $\mathcal{E}$ encloses the information about the conformal structure and Weierstrass data of the marked graph, while the second one is simply translational. The third coordinate has been introduced just for distinguishing between two graphs having $q_{0}=0$ and being symmetric with respect to the plane $\left\{x_{3}=0\right\}$.

The following notation and lemmae are required.

Consider the holomorphic 1-form $\overline{J_{v}^{*}\left(\eta_{j}(v)\right)}$. Taking into account that $J_{v}$ fixes $a_{j}(v)$ pointwise, we infer that $\int_{a_{k}(v)} \overline{J_{v}^{*}\left(\eta_{j}(v)\right)}=\delta_{j k}$, and so, $J_{v}^{*}\left(\eta_{j}(v)\right)=\overline{\eta_{j}(v)}$. Moreover, since $J_{v}\left(b_{j}(v)\right)=-b_{j}(v)$, then $\pi_{j, k}(v)=\int_{b_{k}} \eta_{j}(v)$ is an imaginary number, for any $j$ and $k$.

It follows that there exists a unique analytic mirror involution $I_{v}: \mathcal{J}(v) \rightarrow \mathcal{J}(v)$ satisfying $I_{v}\left(\mathfrak{p}_{v}(w)\right)=\mathfrak{p}_{v}(\bar{w})$, for any $w \in \mathbb{C}^{n}$. Moreover, as $J_{v}(1)=1$ then $\varphi_{v} \circ J_{v}=I_{v} \circ \varphi_{v}$.

We call $\mathcal{I}: \mathcal{J}_{n} \rightarrow \mathcal{J}_{n}$, the map given by $\mathcal{I}\left(v, \mathfrak{p}_{v}(w)\right)=\left(v, I_{v}\left(\mathfrak{p}_{v}(w)\right)\right)$.

Lemma $3.2([8])$ The maps $\varphi: \mathfrak{D i v}_{k} \rightarrow \mathcal{J}_{n}, \varphi(v, D)=\left(v, \varphi_{v}(D)\right)$, and $\hat{T}: \mathcal{T}_{n} \rightarrow \mathcal{J}_{n}$, $\hat{T}(v)=(v, T(v))$ are smooth. 
As a consequence of the smoothness of $\hat{T}$ and $\varphi$ it follows that there are exactly $2^{2 n}$ differentiable maps $\hat{E}_{1}, \ldots, \hat{E}_{2^{2 n}}: \mathcal{T}_{n} \rightarrow \mathcal{J}_{n}, \hat{E}_{j}(v)=\left(v, E_{j}(v)\right)$, satisfying $2 E_{j}(v)=T(v)+$ $\varphi\left(v, 0 \cdot \infty \cdot J_{v}(0) \cdot J_{v}(\infty)\right)$ for any $j$.

The next result shows that these spinor sections are invariant under the mirror involution. This fact will be crucial for recovering the Weierstrass data from an element in the spinorial bundle.

Lemma $3.3 \mathcal{I} \circ \hat{E}_{j}=\hat{E}_{j}$, for any $j=1, \ldots, 2^{2 n}$.

Proof: Indeed, note that $I_{v}\left(E_{j}(v)\right)=E_{j}(v)+\mathfrak{p}_{v}\left(\frac{1}{2} \sum_{h=1}^{n}\left(m_{h}(v) e^{h}+n_{h}(v) \pi^{h}(v)\right)\right)$, where $m_{h}(v), n_{h}(v) \in \mathbb{Z}$ are continuous functions of $v$. Using that $\mathcal{T}_{n}$ is connected we get that $m_{h}(v), n_{h}(v)$ are constant. Hence, the set $\mathcal{A}_{j}:=\left\{v \in \mathcal{T}_{n}: I_{v}\left(E_{j}(v)\right)=E_{j}(v)\right\}$ is either empty or the whole of $\mathcal{T}_{n}$. On the other hand, $E_{j}(v)=E_{1}(v)+q_{j}(v)$, where $2 q_{j}(v)=0$, and so, $I_{v}\left(q_{j}(v)\right)=q_{j}(v)$. Therefore $\mathcal{A}_{1}=\mathcal{T}_{n}$ if and only if $\mathcal{A}_{j}=\mathcal{T}_{n}$ for any $j$.

Consider the compact genus $n$ Riemann surface $N=\left\{(z, w) \in \overline{\mathbb{C}}: w^{2}=\prod_{i=1}^{2 n+2}(z-\right.$ $\left.\left.c_{i}\right)\right\}$, where $c_{i} \in \mathbb{R}$ and $c_{1}<c_{2}<\ldots<c_{2 n}<0<c_{2 n+1}<c_{2 n+2}$. The function $w$ has a well defined branch $w_{+}$on the planar domain $\Sigma=\overline{\mathbb{C}}-\cup_{i=0}^{n}\left[c_{2 i+1}, c_{2 i+2}\right]$. Moreover there exists a biholomorphism from the domain $\left\{\left(z, w_{+}(z)\right): z \in \Sigma\right\} \subset N$ to a circular domain $\Omega\left(v_{0}\right)$, $v_{0} \in \mathcal{T}_{n}$ taking $0_{+}:=\left(0, w_{+}(0)\right)$ to 0 and $\infty_{+}:=\left(\infty, w_{+}(\infty)\right)$ to $\infty$. Furthermore, up to this biholomorphism, $N=N\left(v_{0}\right)$ and $J=J_{v_{0}}$ is given by $J(z, w)=(\bar{z},-\bar{w})$.

Define the meromorphic 1-form $\nu=\prod_{i=1}^{n+1}\left(z-c_{i}\right) \frac{d z}{z w}$ on $N\left(v_{0}\right)$ and observe that its canonical divisor is given by

$$
(\nu)=\frac{c_{1}^{2} \cdot \ldots \cdot c_{n+1}^{2}}{0_{+} \cdot J_{v_{0}}\left(0_{+}\right) \cdot \infty_{+} \cdot J_{v_{0}}\left(\infty_{+}\right)}
$$

where we are identifying $c_{i} \equiv\left(c_{i}, 0\right) \in N\left(v_{0}\right)$. Since $J_{v_{0}}\left(c_{i}\right)=c_{i}$, then $l_{0}:=\sum_{i=1}^{n+1} \varphi_{v_{0}}\left(c_{i}\right) \in$ $\mathcal{J}\left(v_{0}\right)$ is invariant under $I_{v_{0}}$ and $2 l_{0}=T\left(v_{0}\right)+\varphi_{v_{0}}\left(0_{+} \cdot J v_{0}\left(0_{+}\right) \cdot \infty_{+} \cdot J_{v_{0}}\left(\infty_{+}\right)\right)$. Up to relabelling, we can suppose that $l_{0}=E_{1}\left(v_{0}\right)$ and hence $\mathcal{A}_{1}=\mathcal{T}_{n}$. This completes the proof.

Proposition 3.1 The map $\mathcal{E}: \mathfrak{M}_{n} \rightarrow \mathcal{S}_{n} \times \mathbb{L}^{3} /\langle(1,0,0)\rangle \times\{-1,1\}$ is bijective.

Proof: If $x \in \mathcal{S}_{n}, x=(v, D)$, then $\varphi_{v}(D \cdot 0)=E_{i}(v)$ for some $i \in\left\{1, \ldots, 2^{2 n}\right\}$.

Since $I_{v}\left(E_{i}(v)\right)=E_{i}(v)$, (Lemma 3.3) we have $\varphi_{v}(D \cdot 0)-\varphi_{v}\left(J_{v}(D \cdot 0)\right)=0$. By Abel's theorem, there exists a unique meromorphic function $g_{x}^{0}$ of degree $n+1$ on $N(v)$ satisfying

$$
\left(g_{x}^{0}\right)=\frac{D \cdot 0}{J_{v}(D \cdot 0)} \quad \text { and } \quad g_{x}^{0}(1)=1
$$

Observe that since $J_{v}(1)=1$ we have $g_{x}^{0} \circ J_{v}=1 / \overline{g_{x}^{0}}$.

On the other hand, as $\varphi_{v}\left(D \cdot J_{v}(D)\right)-\varphi_{v}\left(J_{v}(\infty) \cdot \infty\right)=T(v)$, then there exists a meromorphic 1-form $\phi$ on $N(v)$ with canonical divisor $\frac{D \cdot J_{v}(D)}{\infty \cdot J_{v}(\infty)}$. Up to a multiplicative constant we can suppose that $\phi$ satisfies $J_{v}^{*}(\phi)=-\bar{\phi}$. If we write $\phi(z)=h(z) \frac{d z}{z-c_{0}(v)}$, 
$z \in U(v)=(\Omega(v)-\{0, \infty\}) \cup\left(\Omega(v)^{*}-\left\{J_{v}(0), J_{v}(\infty)\right\}\right) \cup a_{0}(v)$, we infer that $h(z) \in \mathbb{R}^{*}$, for any $z$ satisfying $\left|z-c_{0}(v)\right|=r_{0}(v)=c_{0}(v)-1$. Then, define

$$
\phi_{3}^{0}(x):=\frac{1}{h(1)} \phi
$$

and observe that the equations

$$
\left(\phi_{3}^{0}(x)\right)=\frac{D \cdot J_{v}(D)}{\infty \cdot J_{v}(\infty)} \quad \text { and } \quad h_{3}^{0}(1)=1
$$

characterize $\phi_{3}^{0}(x)$ as meromorphic 1-form on $N(v)$.

Given $\chi=\left(x, q_{0}, \varepsilon_{0}\right) \in \mathcal{S}_{n} \times \mathbb{L}^{3} /\langle(1,0,0)\rangle \times\{-1,1\}$ it is easy to check that there exist unique $\theta_{\chi} \in\{|z|=1\}$ and $r_{\chi} \in \mathbb{R}$ such that, for $\phi_{3}(\chi)=r_{\chi} \phi_{3}^{0}(x)$ and $g_{\chi}=\theta_{\chi} g_{x}^{0}$, the map $X_{\chi}: \overline{\Omega(v)} \rightarrow \mathbb{L}^{3} /\langle(1,0,0)\rangle$,

$$
X_{\chi}(z):=q_{0}+\text { Real } \int_{1}^{z}\left(\frac{i}{2}\left(\frac{1}{g_{\chi}}-g_{\chi}\right),-\frac{1}{2}\left(\frac{1}{g_{\chi}}+g_{\chi}\right), 1\right) \phi_{3}(\chi)
$$

is well defined and determines a CMF graph $S_{\chi}:=X_{\chi}(\overline{\Omega(v)}-\{0, \infty\}) \in \mathfrak{G}_{n}$ satisfying $X\left(a_{0}(v)\right)=q_{0}$ and $\epsilon_{0}$ is the sign of the third coordinate of the flux along $a_{0}(v)$.

Defining the mark $\mathfrak{m}_{\chi}$ by $q_{j}=X_{\chi}\left(a_{j}(v)\right), i=0, \ldots, n$, it is now clear that $\mathcal{E}^{-1}(\chi)=$ $\left\{\left(S_{\chi}, \mathfrak{m}_{\chi}\right)\right\}$, and so, $\mathcal{E}$ is bijective.

\subsection{Structure of the spinorial bundle $\mathcal{S}_{n}$}

In the previous subsection we have identified $\mathfrak{M}_{n}$ with the space $\mathcal{S}_{n} \times \mathbb{L}^{3} /\langle(1,0,0)\rangle \times\{-1,1\}$. Our aim now is to show that $\mathcal{S}_{n}$, and so $\mathfrak{M}_{n}$, has a natural structure of differentiable manifold.

Theorem 3.1 (Structure of the spinorial bundle) The space $\mathcal{S}_{n}$ is a smooth real ( $3 n+$ 1)-dimensional submanifold of $\mathfrak{D i v}_{n}$ and the map $\mathfrak{v}: \mathcal{S}_{n} \rightarrow \mathcal{T}_{n}, \mathfrak{v}(v, D)=v$ is a finite covering.

Proof: The fact $\mathcal{S}_{n} \neq \emptyset$ follows from the existence of CMSF surfaces with an arbitrary number of singularities in the quotient (see [7]) and Proposition 3.1. The key step of this proof is that $\mathcal{S}_{n}$ does not contain any special divisor (see [6]).

Consider the differentiable map $H: \mathfrak{D i v}_{n} \rightarrow \mathcal{J}_{n}$ given by

$$
H(v, D)=\left(v, 2 \varphi_{v}(D)-\varphi_{v}\left(0 \cdot J_{v}(0) \cdot \infty \cdot J_{v}(\infty)\right)-T(v)\right),
$$

and note that $\mathcal{S}_{n}=\left\{(v, D) \in \mathfrak{D i v}_{n}: H(v, D)=(v, 0)\right\}$. In order to prove that $\mathcal{S}_{n}$ is a differentiable submanifold of $\mathfrak{D i v} \mathfrak{v}_{n}$, it suffices to check that $d H_{q}$ is bijective at any point $q$ of $\mathcal{S}_{n}$. 
Let $q_{0}:=\left(v_{0}, D_{0}\right)$ be an arbitrary point of $\mathcal{S}_{n}$. Observe that $d H_{q_{0}}$ is bijective if and only if the map

$$
\begin{gathered}
H_{0}: \operatorname{Div}_{n}\left(\Omega\left(v_{0}\right)\right) \rightarrow \mathcal{J}\left(v_{0}\right) \\
D \mapsto \varphi_{v_{0}}(D)-\varphi_{v_{0}}\left(D_{0}\right)
\end{gathered}
$$

is a local diffeomorphism at $D_{0}$.

We are going to write the expression of $H_{0}$ in local coordinates around $D_{0} \in \operatorname{Div}_{n}\left(\Omega\left(v_{0}\right)\right)$ and $H_{0}\left(D_{0}\right)=0 \in \mathcal{J}\left(v_{0}\right)$. To do this, write $D_{0}=z_{1}^{m_{1}} \cdot \ldots \cdot z_{s}^{m_{s}} \in \operatorname{Div}_{n}\left(\Omega\left(v_{0}\right)\right)$, and denote by $\left(U_{j}, w_{j}:=z-z_{j}\right)$ the conformal parameter in $\Omega\left(v_{0}\right)$, where $U_{j}$ is the open disc of radius $\epsilon>0$ centered at $z_{j}, j=1, \ldots, s$. Put $U=\prod_{j=1}^{s} U_{j}^{m_{j}} \subset \Omega\left(v_{0}\right)^{n}=\Omega\left(v_{0}\right) \times \stackrel{n}{.} \times \Omega\left(v_{0}\right)$. Then $p_{n}(U)$ is a neighborhood of $D_{0}$ in $\operatorname{Div}_{n}\left(\Omega\left(v_{0}\right)\right)$, where $p_{n}: \Omega\left(v_{0}\right)^{n} \rightarrow \operatorname{Div}_{n}\left(\Omega\left(v_{0}\right)\right)$ is the projection associated to the action of the group of permutations of order $n$ on $\Omega\left(v_{0}\right)^{n}$. A coordinate chart for $\operatorname{Div}_{n}\left(\Omega\left(v_{0}\right)\right)$ around $D_{0}$ is given by

$$
\xi: p_{n}(U) \rightarrow \mathbb{C}^{n}, \quad \xi\left(\prod_{j=1}^{s} Q_{1, m_{j}} \cdot \ldots \cdot Q_{m_{j}, m_{j}}\right)=\left(\left(t_{1, m_{j}}, \ldots, t_{m_{j}, m_{j}}\right)_{j=1, \ldots, s}\right)
$$

where $t_{h, m_{j}}=\sum_{l=1}^{m_{j}}\left(z_{j}\left(Q_{l, m_{j}}\right)\right)^{h}, h=1, \ldots, m_{j}, j=1, \ldots, s$. For more details see [6].

Label $\mathfrak{p}: \mathbb{C}^{n} \rightarrow \mathcal{J}\left(v_{0}\right)=\mathbb{C}^{n} / L\left(v_{0}\right)$ as the natural projection and consider a neighborhood $W^{\prime}$ of $H\left(D_{0}\right)=0$ such that $\mathfrak{p}: W:=\mathfrak{p}^{-1}(W) \rightarrow W^{\prime}$ is a diffeomorphism and $H_{0}(U) \subset W^{\prime}$.

Write $\eta_{i}\left(v_{0}\right)\left(w_{j}\right)=f_{i, j}\left(w_{j}\right) d w_{j}$ on $W_{j}:=w_{j}\left(U_{j}\right)$ for $i=1, \ldots, n, j=1, \ldots s$. The local expression $\hat{H}_{0}$ of $H_{0}$ around $D_{0}, \hat{H}_{0}=\mathfrak{p}^{-1} \circ H_{0} \circ \xi^{-1}$, is given by

$$
\begin{gathered}
\hat{H}_{0}: \xi\left(p_{n}(U)\right) \rightarrow W \\
\hat{H}_{0}(t)=\sum_{j=1}^{s} \sum_{h=1}^{m_{j}} \int_{0}^{w_{h, m_{j}}} f_{j}\left(w_{j}\right) d w_{j}
\end{gathered}
$$

where $f_{j}={ }^{T}\left(f_{1, j}, \ldots, f_{n, j}\right), w_{h, m_{j}} \equiv w_{j}, t_{l, m_{j}}=\sum_{h=1}^{m_{j}} w_{h, m_{j}}^{l}, h=1, \ldots, m_{j}, t=\left(t_{1, m_{j}}, \ldots, t_{m_{j}, m_{j}}\right)_{j=1, \ldots, s}$.

Put $f_{j}\left(w_{j}\right)=\sum_{l=0}^{\infty} b_{j, l} w_{j}^{l}, b_{j, l} \in \mathbb{C}^{n}, j=1 \ldots s$. Then the Taylor series for the holomorphic map $w_{h, m_{j}} \mapsto \int_{0}^{w_{h, m_{j}}} f_{j}\left(w_{j}\right) d w_{j}$ is $\int_{0}^{w_{h, m_{j}}} f_{j}\left(w_{j}\right) d w_{j}=\sum_{l=1}^{\infty} a_{j, l} w_{h, m_{j}}^{l}$, where $a_{j, l}=$ $\frac{1}{l} b_{j, l-1}, l \geq 1, j=1, \ldots, s$. It is not hard to check that $\hat{H}_{0}(t) \sum_{j=1}^{s} \sum_{l=1}^{m_{j}} a_{j, l} t_{l, m_{j}}+R(t)$, where the first derivatives of $R$ with respect to $t_{l, m_{j}}$ vanish at $t=0$, and so the column vectors of the Jacobian matrix of $\hat{H}_{0}$ are $\left\{a_{l, j}, l=1, \ldots, m_{j}, j=1 \ldots, s\right\}$.

Reasoning by contradiction, suppose that the rows of that matrix are linearly dependent, which is equivalent to saying that there exists a holomorphic 1-form $\omega_{0}$ in $N\left(v_{0}\right)$ having a zero at $z_{j} \in \Omega\left(v_{0}\right) \subset N\left(v_{0}\right)$ of order at least $m_{j}, j=1, \ldots, s$. A direct application of Riemann-Roch theorem gives the existence of a non-constant meromorphic function $f$ on $N\left(v_{0}\right)$ having poles at $z_{1}, \ldots, z_{s}$ with order at most $m_{1}, \ldots, m_{s}$, respectively. In particular, $f$ has degree less than or equal to $n$. As $J_{v_{0}}(0)$ is not a pole of $f$, up to adding a constant we can suppose that $f\left(J_{v_{0}}(0)\right)=0$. 
On the other hand, since $\varphi_{v_{0}}\left(D_{0}\right)+\varphi_{v_{0}}(0)=E_{i}\left(v_{0}\right)$ and $I_{v_{0}}\left(E_{i}\left(v_{0}\right)\right)=E_{i}\left(v_{0}\right), i \in$ $\left\{1, \ldots, 2^{2 n}\right\}$, then we get $\varphi_{v_{0}}\left(D_{0} \cdot 0\right)-\varphi_{v_{0}}\left(J_{v_{0}}\left(D_{0} \cdot 0\right)\right)=0$. Therefore, a direct application of Abel's theorem gives the existence of a meromorphic function $g$ of degree $n+1$ on $N\left(v_{0}\right)$ whose principal divisor coincides with $\frac{D_{0} \cdot 0}{J_{v_{0}}\left(D_{0} \cdot 0\right)}$. As $J_{v_{0}}$ is an antiholomorphic involution with fixed points, it is not hard to check that $g \circ J_{v_{0}}=r / \bar{g}, r>0$. Hence, up to multiplying $g$ by the factor $r^{-1 / 2}$, we can suppose that $g \circ J_{v_{0}}=1 / \bar{g}$.

Note that $g \in \mathcal{F}_{v_{0}}$, where for any $v \in \mathcal{T}_{n}, \mathcal{F}_{v}$ denotes the family of meromorphic functions $h$ of degree $n+1$ in $N(v)$ with zeroes in $\Omega(v)$ and satisfying $h \circ J_{v}=1 / \bar{h}$.

Claim: Let $f_{\lambda}$ denote the meromorphic function $\frac{1+\lambda f}{1+\overline{\lambda\left(f \circ J_{v_{0}}\right)}}, \lambda \in \mathbb{C}$. Then, $f_{\lambda}$ is not constant, for any $\lambda \in \mathbb{C}^{*}$. Moreover, $g_{\lambda}:=g f_{\lambda} \in \mathcal{F}_{v_{0}}$ for any $\lambda \in \mathbb{C}$.

Assume $f_{\lambda}=c$, where $c, \lambda \in \mathbb{C}^{*}$. Then, we infer that $1+\lambda f=c\left(1+\overline{\lambda\left(f \circ J_{v_{0}}\right)}\right)$ and so the polar divisor of $f$, which is contained in $D_{0}$, is invariant under $J_{v_{0}}$. This is absurd because $D_{0} \in \operatorname{Div}_{n}\left(\Omega\left(v_{0}\right)\right)$ and $\Omega\left(v_{0}\right) \cap J_{v_{0}}\left(\Omega\left(v_{0}\right)\right)=\emptyset$.

For the second part of the claim, first note that the principal divisor of $g_{\lambda}$ is $\left(g_{\lambda}\right)=$ $\frac{D_{\lambda} \cdot \infty}{J_{v_{0}}\left(D_{\lambda}\right) \cdot J_{v_{0}}(\infty)}$, where $D_{\lambda}$ is an integral divisor of degree $\leq n$ and so the degree of $g_{\lambda}$ is $\leq n+1, \lambda \in \mathbb{C}$. Moreover, $g_{\lambda}$ is not constant for any $\lambda$ (otherwise, $J_{v_{0}}(0)$ would be a zero of $1+\lambda f$, contradicting $\left.f\left(J_{v_{0}}(0)\right)=0\right)$.

Let $A$ be the set $\left\{\lambda \in \mathbb{C}: g_{\lambda} \in \mathcal{F}_{v_{0}}\right\}$, and observe that $0 \in A$. It suffices to see that $A$ is open and closed.

The openness of $A$ is an elementary consequence of Hurwitz theorem (we are using the fact that the degree of $g_{\lambda}$ is at most $n+1$ ).

Finally, let us prove that $A$ is closed. Let $\lambda_{0} \in \bar{A}$, and take $\left\{\lambda_{n}\right\}_{n \in \mathbb{N}} \rightarrow \lambda_{0}$, where $\left\{\lambda_{n}: n \in \mathbb{N}\right\} \subset A$. The sequence $\left\{g_{n}:=g_{\lambda_{n}}\right\}_{n \in \mathbb{N}}$ converges to $g_{0}:=g_{\lambda_{0}}$ uniformly on $N\left(v_{0}\right)$. We know that $g_{n} \circ J_{v_{0}}=1 / \overline{g_{n}}$ and so the zeros of $g_{n}$ lie in $\Omega\left(v_{0}\right)$, therefore, $g_{n}$ is holomorphic on $\Omega\left(v_{0}\right), n \in \mathbb{N}$ and so the same holds for $g_{0}$. Moreover, since $\left|g_{0}\right|=1$ on $\partial \Omega\left(v_{0}\right)$ and it is non constant, the maximum principle implies that $\left|g_{0}\right|<1$ on $\Omega\left(v_{0}\right)$ and we infer that $g_{0}$ has no critical points on $\partial \Omega_{v_{0}}$. As $\partial \Omega_{v_{0}}$ consists of $n+1$ disjoint circles, this means that $g_{0}$ takes on any complex number $\theta \in \mathbb{S}^{1}$ at least $n+1$ times. Hence the degree of $g_{0}$ must be $n+1$ and $g_{0} \in \mathcal{F}_{v_{0}}$. This concludes the proof of the claim.

To get the desired contradiction take $P \in \partial \Omega\left(v_{0}\right)$ such that $f(P) \neq 0, \infty$, and choose $\lambda^{\prime}=\frac{-1}{f(P)}$. Since $J_{v_{0}}(P)=P$, the meromorphic function $g_{\lambda^{\prime}}$ has degree less than $n+1$, and so, $\lambda^{\prime} \notin A=\mathbb{C}$, which is absurd.

Summarizing, we have proved that $\left.H\right|_{\mathcal{S}_{n}}: \mathcal{S}_{n} \rightarrow \mathbf{0}, H(v, D)=(v, 0)$, is a local diffeomorphism, where $\mathbf{0}=\left\{(v, 0): v \in \mathcal{T}_{n}\right\} \subset \mathcal{J}_{n}$ is the null section in the Jacobian bundle. Consequently, the projection $\mathfrak{v}: \mathcal{S}_{n} \rightarrow \mathcal{T}_{n}, \mathfrak{v}(v, D)=v$, is a local diffeomorphism too. To finish, it suffices to check that $\mathfrak{v}$ is also proper. Indeed, take a sequence $\left\{\left(v_{k}, D_{k}\right)\right\}_{k \in \mathbb{N}} \subset \mathcal{S}_{n}$ such that $\left\{v_{k}\right\}_{k \in \mathbb{N}}$ converges to a point $v_{\infty} \in \mathcal{T}_{n}$. We can assume that $\varphi_{v_{k}}\left(D_{k} \cdot 0\right)=E_{i}\left(v_{k}\right)$ for any $k \in \mathbb{N}$. Since $I_{v_{k}}\left(E_{i}\left(v_{k}\right)\right)=E_{i}\left(v_{k}\right)$, we get $\varphi_{v_{k}}\left(D_{k} \cdot 0\right)-\varphi_{v_{k}}\left(J_{v_{k}}\left(D_{k} \cdot 0\right)\right)=0$. By Abel's theorem there is a meromorphic function $g_{k} \in \mathcal{F}_{v_{k}}$ with canonical divisor $\frac{D_{k} \cdot 0}{J_{v_{k}}\left(D_{k} \cdot 0\right)}$. 
Let us see that, up to taking a subsequence, $\left\{g_{k}\right\}_{k \in \mathbb{N}} \rightarrow g_{\infty} \in \mathcal{F}_{v_{\infty}}$. Reflecting about all the components of $\partial \Omega\left(v_{k}\right)$, we can meromorphically extend $g_{k}$ to a planar open neighborhood $W_{k}$ of $\overline{\left.\Omega\left(v_{k}\right)\right)}, k \in \mathbb{N}$. By continuity and for $k_{0}$ large enough, the set $W=\cap_{k \geq k_{0}} W_{k}$ is a planar neighborhood of $\overline{\Omega\left(v_{\infty}\right)}$. Classical normality criteria show that, up to taking a subsequence, $\left\{g_{k}\right\}_{k \in \mathbb{N}}$ converges uniformly on $\overline{\Omega\left(v_{\infty}\right)}$ to a function $g_{\infty}$ which is meromorphic beyond $\overline{\Omega\left(v_{\infty}\right)}$. It is clear that $\left|g_{\infty}\right|=1$ on $\partial \Omega\left(v_{\infty}\right),\left|g_{\infty}\right|<1$ on $\Omega\left(v_{\infty}\right)$ and $g_{\infty}(0)=0$. This proves that $g_{\infty}$ is non constant and can be extended to $N\left(v_{\infty}\right)$ by the Schwarz reflection $g_{\infty} \circ J_{\infty}=1 / \overline{g_{\infty}}$. Since $\operatorname{deg}\left(g_{k}\right)=n+1$, then Hurwitz theorem implies that $\operatorname{deg}\left(g_{\infty}\right) \leq n+1$. On the other hand, $\left|g_{\infty}\right|=1$ only on $\partial \Omega\left(v_{\infty}\right)$, and so $g_{\infty}$ is injective on every boundary component of $\Omega\left(v_{\infty}\right)$. Therefore, the degree of $g_{\infty}$ must be exactly $n+1$ and $g_{\infty} \in \mathcal{F}_{v_{\infty}}$.

Finally, note that $\frac{D_{\infty} \cdot 0}{J_{v_{\infty}}\left(D_{\infty} \cdot 0\right)}$ where $D_{\infty} \in \mathfrak{D i v}_{n}$ and use Hurwitz theorem to infer that $\left\{D_{k}\right\}_{k \in \mathbb{N}} \rightarrow D_{\infty} \in \mathfrak{D i v}_{n}$. Since $\mathcal{S}_{n}$ is a closed subset of $\mathfrak{D i v _ { n }}$, we get $D_{\infty} \in \mathcal{S}_{n}(i)$, which proves the properness of $\mathfrak{v}: \mathcal{S}_{n} \rightarrow \mathcal{T}_{n}$ and so the theorem.

\subsection{Proof of the Main Theorem}

In the preceding section, we have endowed $\mathfrak{M}_{n}$ of a differentiable structure. It is natural to ask whether $\mathfrak{s}_{2}: \mathfrak{M}_{n} \rightarrow \mathbb{R}^{3 n+4}$ is a smooth map or not.

In order to do this, we have to show that the Weierstrass data of elements in $\mathfrak{M}_{n}$ depends smoothly on its associated divisor in $\mathcal{S}_{n}$. This requires a convenient concept of differentiability for maps from the bundle of divisors to the space of meromorphic functions or 1-forms. The first part of this subsection is devoted to present these concepts.

For any $v \in \mathcal{T}_{n}$, call $\mathcal{M}(v)$ the family of meromorphic functions on $N(v)$. The corresponding bundle over $\mathcal{T}_{n}$ is denoted by $\mathcal{M}_{n}=\cup_{v \in \mathcal{T}_{n}} \mathcal{M}(v)$.

Likewise, we call $\mathcal{H}(v)$ the space of meromorphic 1-forms on $N(v)$ and denote by $\mathcal{H}_{n}=\cup_{v \in \mathcal{T}_{n}} \mathcal{H}(v)$ the associated bundle over $\mathcal{T}_{n}$.

Given $v \in \mathcal{T}_{n}$ and $k_{1}, k_{2} \in \mathbb{N}$ we denote by $\operatorname{Div}_{k_{1}, k_{2}}(v)$ the product manifold,

$$
\operatorname{Div}_{k_{1}, k_{2}}(v)=\operatorname{Div}_{k_{1}}(v) \times \operatorname{Div}_{k_{2}}(v),
$$

and by $\mathfrak{D i v}_{k_{1}, k_{2}}$ its associated bundle over $\mathcal{T}_{n}, \mathfrak{D i v}_{k_{1}, k_{2}}=\cup_{v \in \mathcal{T}_{n}} D i v_{k_{1}, k_{2}}(v)$. Like in the case of $\mathfrak{D i v}_{k}, \mathfrak{D i v}_{k_{1}, k_{2}}$ has a natural structure of analytical manifold. We use the convention $\mathfrak{D i v}_{k, 0}=\mathfrak{D i v} \mathfrak{v}_{k}$ and $\mathfrak{D i v} \mathfrak{v}_{0,0}=\mathcal{T}_{n}$.

Definition 3.3 (smoothness with $k$-regularity) Let $M_{j}$ be a real manifold of dimension $m_{j}, j=1,2,3$, and let $f: M_{1} \times M_{2} \rightarrow M_{3}$ be a $\mathcal{C}^{k}$ map. The map $f$ is said to be differentiable (or smooth) with $k$-regularity in $M_{1}$ if, for any charts $\left(U_{1} \times U_{2},(x \equiv\right.$ $\left.\left.\left(x_{1}, \ldots, x_{m_{1}}\right), y \equiv\left(y_{1}, \ldots, y_{m_{2}}\right)\right)\right)$ in $M_{1} \times M_{2}$ and $\left(U_{3}, z \equiv\left(z_{1}, \ldots, z_{m_{3}}\right)\right)$ in $M_{3}$, the local 
expression of $f, f(x, y): x\left(U_{1}\right) \times y\left(U_{2}\right) \rightarrow z\left(U_{3}\right)$, satisfies that $f(\cdot, y)$ is smooth in $x\left(U_{1}\right)$ for any $y \in y\left(U_{2}\right)$, and all the partial derivatives of $f(x, y)$ with respect to variables in $x$ are $\mathcal{C}^{k}$ in $x\left(U_{1}\right) \times y\left(U_{2}\right)$.

Definition 3.4 (smooth deformation of the double of a circular domain) Let $v_{0} \in$ $\mathcal{T}_{n}$ and $\epsilon>0$ small enough. Denote by $V(\epsilon)$ the Euclidean ball of radius $\epsilon$ in $\mathcal{T}_{n}$ centered at $v_{0}$. Since $V(\epsilon)$ is simply connected, standard homotopy arguments in differential topology show the existence of a family of diffeomorphisms $\left\{F_{v}: N\left(v_{0}\right) \rightarrow N(v): v \in V(\epsilon)\right\}$ such that $F_{v_{0}}=I d, F_{v}(\infty)=\infty, J_{v} \circ F_{v} \circ J_{v_{0}}=F_{v}$, for any $v \in V(\epsilon)$, and $F: V(\epsilon) \times \overline{\Omega\left(v_{0}\right)} \rightarrow \mathbb{C}$, $F(v, z):=F_{v}(z)$, is smooth.

By definition, we say that $\left\{F_{v}: N\left(v_{0}\right) \rightarrow N(v): v \in V(\epsilon)\right\}$ is a smooth deformation of $N\left(v_{0}\right)$. Moreover note that, for $\epsilon$ small enough, $\frac{\partial F}{\partial z} \neq 0$ in $V(\epsilon) \times \overline{\Omega\left(v_{0}\right)}$.

Let $W \subset \mathfrak{D i v}_{k_{1}, k_{2}}$ be a submanifold, and let $h: W \rightarrow \mathcal{M}_{n}$ be a map preserving the fibers, that is to say, $h_{v, D_{1}, D_{2}}:=h\left(v, D_{1}, D_{2}\right) \in \mathcal{M}(v)$ for any $\left(v, D_{1}, D_{2}\right) \in W$. We are going to define the notion of differentiability with $k$-regularity of $h$. Take $\mathcal{V}$ any coordinate neighborhood in $\mathfrak{D i v}_{k_{1}, k_{2}}$ meeting $W$. Denote by $V$ the associated neighborhood to $\mathcal{V}$ in $\mathcal{T}_{n}$ and call $v_{0} \in V$ an interior point. Take a smooth deformation of $N\left(v_{0}\right),\left\{F_{v}: N\left(v_{0}\right) \rightarrow\right.$ $N(v): v \in V(\epsilon)\}$. We say that $h$ is differentiable with $k$-regularity in $\mathcal{V} \cap W$ if the map

$$
\begin{gathered}
\hat{h}:(\mathcal{V} \cap W) \times N\left(v_{0}\right) \rightarrow \overline{\mathbb{C}}, \\
\hat{h}\left(\left(v, D_{1}, D_{2}\right), x\right)=h_{v, D_{1}, D_{2}}\left(F_{v}(x)\right)
\end{gathered}
$$

is smooth with $k$-regularity in $\mathcal{V} \cap W$. The map $h$ is said to be differentiable with $k$ regularity on $W$ if it does in $\mathcal{V} \cap W$, for any coordinate neighborhood $\mathcal{V}$ meeting $W$. It is easy to check that this definition does not depend on choice of neither $v_{0}$ nor the smooth deformation of $N\left(v_{0}\right)$.

Likewise, for a map $\omega: W \rightarrow \mathcal{H}_{n}$ preserving the fibers, define $\hat{\omega}: \mathcal{V}(\epsilon) \cap W \rightarrow \mathcal{H}\left(v_{0}\right)$ by

$$
\hat{\omega}\left(v, D_{1}, D_{2}\right)=\left(F_{v}^{*}\left(\omega_{v, D_{1}, D_{2}}\right)\right)^{(1,0)}
$$

where the superscript $(1,0)$ means the $(1,0)$ part of the 1 -form (by definition $(f d z+$ $\left.g d \bar{z})^{(1,0)}=f d z\right)$. We say that $\omega$ is differentiable with $k$-regularity in $\mathcal{V}(\epsilon) \cap W$ if for any local chart $(U, z)$ in $N\left(v_{0}\right)$, the map $\hat{f}:(\mathcal{V}(\epsilon) \cap W) \times U \rightarrow \overline{\mathbb{C}}$, given by $\hat{f}\left(\left(v, D_{1}, D_{2}\right), z\right)=$ $\hat{\omega}\left(v, D_{1}, D_{2}\right)(z) / d z$ is smooth with $k$-regularity in $\mathcal{V}(\epsilon) \cap W$. The global concept of differentiability with $k$-regularity in $W$ is defined in the obvious way.

The following 1-forms we will play an important role during the proof of the Main Theorem.

Given $D=\prod_{j=1}^{s} w_{j}^{m_{j}} \in \operatorname{Div}_{k}(\Omega(v))$, we denote by $\tau_{D}(v)$ the unique meromorphic 1-form on $N(v)$ satisfying:

- $\tau_{D}(v)$ has simple poles at $w_{j}$ and $J_{v}\left(w_{j}\right), j=1, \ldots, s$, and no other poles,

- $\operatorname{Residue}_{w_{j}}\left(\tau_{D}(v)\right)=-\operatorname{Residue}_{J_{v}\left(w_{j}\right)}\left(\tau_{D}(v)\right)=-m_{j}$ for any $j$ 
- $\int_{a_{i}(v)} \tau_{D}(v)=0$, for any $i=1, \ldots, n$.

Likewise, take $D_{1}=\prod_{j=1}^{s} w_{j, 1}^{m_{j}}, D_{2}=\prod_{h=1}^{r} w_{h, 2}^{n_{h}} \in \operatorname{Div}_{k}(\Omega(v))$ and define $\kappa_{D_{1}, D_{2}}(v)$ as the unique meromorphic 1 -form on $N(v)$ satisfying

- $\kappa_{D_{1}, D_{2}}(v)$ has simple poles at $w_{j, 1}, w_{h, 2}$ and $J_{v}\left(w_{j, 1}\right), J_{v}\left(w_{h, 2}(v), j=1, \ldots, s, h=\right.$ $1, \ldots, r$, and no other poles,

- $\operatorname{Residue}_{w_{j, 1}}\left(\kappa_{D_{1}, D_{2}}(v)\right)=\operatorname{Residue}_{J_{v}\left(w_{j, 1}\right)}\left(\kappa_{D_{1}, D_{2}}(v)\right)=-m_{j}$, for any $j$

- $\operatorname{Residue}_{w_{h, 2}}\left(\kappa_{D_{1}, D_{2}}(v)\right)=$ Residue $_{J_{v}\left(w_{h, 2}\right)}\left(\kappa_{D_{1}, D_{2}}(v)\right)=n_{h}$ for any $h$

- $\int_{a_{i}(v)} \kappa_{D_{1}, D_{2}}(v)=0$, for any $i=1, \ldots, n$.

Lemma $3.4([8])$ The maps $\eta_{j}: \mathcal{T}_{n} \rightarrow \mathcal{H}_{n}, v \mapsto \eta_{j}(v), \tau: \mathfrak{D i v}_{k} \rightarrow \mathcal{H}_{n},(v, D) \mapsto \tau_{D}(v)$, and $\kappa: \mathfrak{D i v}_{k, k} \rightarrow \mathcal{H}_{n},\left(v, D_{1}, D_{2}\right) \mapsto \kappa_{D_{1}, D_{2}}(v)$ are differentiable with 1-regularity.

As a consequence, the functions $\pi_{j, k}(v):=\int_{b_{j}(v)} \eta_{k}(v)$, are differentiable on $\mathcal{T}_{n}$.

The following theorem will show that $\mathfrak{M}_{n}$ and $\mathfrak{G}_{n}$ are analytic manifolds of dimension $3 n+4$. We first need the following lemma, proved in [8]:

Lemma 3.5 ([8]) Given $v \in \mathcal{T}_{n}$, there exists a holomorphic 1-form $\omega_{0}$ in $N(v)$ having $2 n-2$ distinct zeroes, none of them contained in $\partial \Omega(v)$, and satisfying $J_{v}^{*}\left(\omega_{0}\right)=\overline{\omega_{0}}$.

Theorem 3.2 (Main theorem) The map

$$
\left.\mathfrak{s}_{2}: \mathfrak{M}_{n} \rightarrow\left(\mathbb{L}^{3} /\langle(1,0,0)\rangle\right)^{n+1} \times\right]-1,1\left[, \quad \mathfrak{s}_{2}(G, \mathfrak{m})=(\mathfrak{m}, c),\right.
$$

where $c$ is the normal direction at the non-normalized end, is injective and smooth. Hence, $\mathfrak{s}_{2}\left(\mathfrak{M}_{n}\right)$ is open and so $\mathfrak{s}_{2}$ provides a global system of analytic coordinates on $\mathfrak{M}_{n}$.

Moreover, the action of the group of permutations of order $n+1, \mu: \mathfrak{M}_{n} \times \mathcal{P}_{n} \rightarrow \mathfrak{M}_{n}$, is discontinuous. Hence the orbit space, naturally identified to $\mathfrak{G}_{n}$, has a unique analytic structure making $\mathfrak{s}_{1}: \mathfrak{M}_{n} \rightarrow \mathfrak{G}_{n}, \mathfrak{s}_{1}(G, \mathfrak{m})=G$, an analytic covering of $(n+1)$ ! sheets.

Proof: To see that $\mathfrak{s}_{2}$ is one to one, suppose $\left(G_{i}, \mathfrak{m}_{i}\right) \in \mathfrak{M}_{n}, i=1,2$ satisfy $\mathfrak{s}_{1}\left(G_{1}, \mathfrak{m}_{1}\right)=$ $\mathfrak{s}_{1}\left(G_{2}, \mathfrak{m}_{2}\right)=(\mathfrak{m}, c)$. From our normalizations, one end of both of them is asymptotic to $\left\{x_{3}=0, x_{2} \geq 0\right\}$. Since $G_{1}$ and $G_{2}$ are graphs over $\left\{x_{3}=0\right\} \subset \mathbb{L}^{3} /\langle(1,0,0)\rangle$ it follows that, for both surfaces, the other end is asymptotic to $\Pi \cap\left\{x_{2} \leq 0\right\}$, where $\Pi$ is the plane determined by the normal direction $c$. Therefore, $G_{1}$ and $G_{2}$ are contained in a common horizontal half space and by Theorem 2.3 we get $G_{1}=G_{2}$.

To finish the first part of the theorem, it is enough to check that $\mathfrak{s}_{2}$ is smooth and then use the Domain Invariance Theorem. Here we have endowed $\mathfrak{M}_{n}$ with the differentiable structure induced by $\mathcal{E}: \mathfrak{M}_{n} \rightarrow \mathcal{S}_{n} \times \mathbb{L}^{3} /\langle(1,0,0)\rangle \times\{1,-1\}$ (see Definition 3.2).

Let $(S, \mathfrak{m}) \in \mathfrak{M}_{n}$ and label $\chi=\left(x, q_{0}, \varepsilon_{0}\right)=\mathcal{E}(S, \mathfrak{m})$. Following the notation in the proof of the proposition [3.1, call $X_{\chi}$ the associated maximal immersion and label as $\left(g_{\chi}, \phi_{3}(\chi)\right)$ its Weierstrass data. 


\section{Claim The maps}

$$
\begin{aligned}
& \mathcal{S}_{n} \times \mathbb{L}^{3} /\langle(1,0,0)\rangle \times\{1,-1\} \rightarrow \mathcal{M}_{n}, \quad \text { and } \mathcal{S}_{n} \times \mathbb{L}^{3} /\langle(1,0,0)\rangle \times\{1,-1\} \rightarrow \mathcal{H}_{n}, \\
& \chi \mapsto g_{\chi} \quad \chi \mapsto \phi_{3}(\chi)
\end{aligned}
$$

are smooth with 2-regularity and 1-regularity, respectively. Consequently, the map $\mathfrak{s}_{2}$ is smooth.

First we prove that the maps $\mathcal{S}_{n} \rightarrow \mathcal{M}_{n}, x \mapsto g_{x}^{0}$, and $\mathcal{S}_{n} \rightarrow \mathcal{H}_{n}, x \mapsto \phi_{3}^{0}(x)$, given by equations (2) and (3), are smooth with 2-regularity and 1-regularity, respectively.

Indeed, take $x_{0}=\left(v_{0}, D_{0}\right) \in \mathcal{S}_{n}$. From Theorem 3.1, there exists an open ball $V(\epsilon)$ in $\mathcal{T}_{n}$ centered at $v_{0}$ of radius $\epsilon>0$ and a local diffeomorphism $V(\epsilon) \rightarrow \mathcal{S}_{n}, v \mapsto(v, D(v))$, where $D\left(v_{0}\right)=D_{0}$. We label $\mathcal{V}(\epsilon)$ as the image of $V(\epsilon)$ under this map. For simplicity, we write $x(v):=(v, D(v)), v \in V(\epsilon)$.

Therefore, the map $V(\epsilon) \rightarrow \mathfrak{D i v}_{n+1}, v \rightarrow(v, D(v) \cdot 0)$ is smooth, and since $\tau: \mathfrak{D i v}_{n+1} \rightarrow$ $\mathcal{H}_{n}$ is also smooth with 1-regularity (see Corollary 3.4), the same holds for the map $V(\epsilon) \rightarrow$ $\mathcal{H}_{n}, v \mapsto \tau_{v}:=\tau_{D(v) \cdot 0}(v)$

Take a smooth deformation of $N\left(v_{0}\right),\left\{F_{v}: N\left(v_{0}\right) \rightarrow N(v): v \in V(\epsilon)\right\}$. Let $B\left(v_{0}\right)=$ $\left\{a_{1}\left(v_{0}\right), \ldots, a_{n}\left(v_{0}\right), b_{1}\left(v_{0}\right), \ldots, b_{n}\left(v_{0}\right)\right\}$ be the canonical homology basis on $\Omega\left(v_{0}\right)$ defined as in Subsection 3.1. Observe that $N\left(v_{0}\right)-\cup_{j=1}^{n}\left(a_{j}\left(v_{0}\right) \cup b_{j}\left(v_{0}\right)\right)$ is simply connected, moreover, without loss of generality we can suppose that this domain does not contains the points in $D_{0} \cdot 0$. For $v$ close enough to $v_{0}$ the curves $a_{j}(v):=F_{v}\left(a_{j}\left(v_{0}\right)\right), b_{j}\left(v_{0}\right):=F_{v}\left(b_{j}\left(v_{0}\right)\right)$ are a canonical basis of $N(v)$ and do not pass also through the points in $D(v) \cdot 0, j=1, \ldots, n$.

By Abel's theorem, and for $z \in N(v)-\cup_{j=1}^{n}\left(a_{j}(v) \cup b_{j}(v)\right)$ we have

$$
g_{x(v)}^{0}(z)=\operatorname{Exp}\left(\int_{1}^{z}\left(\tau_{v}+\sum_{j=1}^{n} m_{j}(v) \eta_{j}(v)\right)\right)
$$

In this expresion, the integration paths lie in $\left(N(v)-\cup_{j=1}^{n}\left(a_{j}(v) \cup b_{j}(v)\right)\right) \cup\{1\}$, and $m_{j}(v) \in \mathbb{Z}$ are integer numbers determined by the equation:

$$
\widetilde{\varphi_{v}}(D(v) \cdot 0)-\widetilde{\varphi_{v}}\left(J_{v}(0) \cdot J_{v}(D(v))\right)=\sum_{j=1}^{n} m_{j}(v) \pi^{j}(v),
$$

where $\widetilde{\varphi_{v}}$ is the branch of $\varphi_{v}$ on $N(v)-\cup_{j=1}^{n}\left(a_{j}(v) \cup b_{j}(v)\right)$ vanishing at 1 .

Since $m_{j}(v)$ depend continuously on $v$, then $m_{j}(v)=m_{j} \in \mathbb{Z}$ and so, by Corollary [3.4. $g_{x(v)}^{0}$ depends smoothly on $v$ with 2-regularity.

We have to obtain the analogous result for the map $\mathcal{V}(\epsilon) \rightarrow \mathcal{H}_{n}, v \rightarrow \phi_{3}^{0}(x(v))$. Take the holomorphic 1-form $\omega_{0}$ on $N\left(v_{0}\right)$ given in Lemma 3.5, write $\nu\left(v_{0}\right):=\omega_{0}=\sum_{j=1}^{n} \lambda_{j} \eta_{j}\left(v_{0}\right)$, where $\lambda_{j} \in \mathbb{R}$, and define $\nu(v):=\sum_{j=1}^{n} \lambda_{j} \eta_{j}(v)$. Since the map $v \mapsto \nu(v)$ is smooth with 1-regularity (see Corollary 3.4) it suffices to prove that $v \mapsto \frac{\phi_{3}^{0}(x(v))}{\nu(v)}$ is smooth with 2-regularity. 
By Hurwitz's Theorem and the implicit function theorem, $\nu(v)$ satisfies also the thesis in Lemma 3.5. for $v \in V(\epsilon)$, for $\epsilon>0$ small enough. Moreover, as explained during the proof of Lemma 3.5 the map $V(\epsilon) \rightarrow \mathfrak{D i v}_{2 n-2}, v \mapsto(v,(\nu(v)))$ is at least $\mathcal{C}^{1}$, where as usually $(\nu(v))$ is the canonical divisor associated to $\nu(v)$. Hence, writing $(\nu(v))=A_{v} \cdot J_{v}\left(A_{v}\right)$, the map $V(\epsilon) \rightarrow \mathfrak{D i v}_{n-1}, v \mapsto\left(v, A_{v}\right)$, is also smooth, and therefore, the same holds for $V(\epsilon) \rightarrow \mathfrak{D i v}_{n, n}, v \mapsto\left(v, D(v), \infty \cdot A_{v}\right)$. We infer from Corollary 3.4 that the map $V(\epsilon) \rightarrow \mathcal{H}_{n}$, $v \mapsto \kappa_{v}:=\kappa_{\infty \cdot A_{v}, D(v)}(v)$, is smooth with 1-regularity. Reasoning as above, the map

$$
f_{x(v)}(z)=\operatorname{Exp}\left(\int_{1}^{z}\left(\kappa_{v}+\sum_{j=1}^{n} n_{j} \eta_{j}(v)\right)\right)
$$

is a well defined meromorphic function on $N(v)$, for suitable integer numbers $n_{j}$ not depending on $v$ and $V(\epsilon) \mathfrak{D i v}_{n} \rightarrow \mathcal{M}_{n}, v \mapsto f_{x(v)}$, is smooth with 2-regularity. The principal divisor associated to $f_{x(v)}$ is given by $\left(f_{x(v)}\right)=\frac{D(v) \cdot J_{v}(D(v))}{\infty \cdot A_{v} \cdot J_{v}(\infty) \cdot J_{v}\left(A_{v}\right)}$. Therefore, if we write $\nu(v)=h_{v}(z) \frac{d z}{z}$ on $U(v)=(\Omega(v)-\{0, \infty\}) \cup\left(\Omega(v)^{*}-\left\{J_{v}(0), J_{v}(\infty)\right\}\right) \cup a_{0}(v)$, we infer that $\frac{\phi_{3}^{0}(x(v))}{\nu(v)}=\frac{1}{h_{v}(1)} f_{x(v)}$, and so $v \mapsto \phi_{3}^{0}(x(v))$ is smooth with 1-regularity.

It follows that the map

$$
X_{x}^{0}:=\operatorname{Real} \int_{1} \Phi(x)^{0} \quad \Phi(x)^{0}:=\left(\frac{i}{2}\left(\frac{1}{g_{x}^{0}}-g_{x}^{0}\right),-\frac{1}{2}\left(\frac{1}{g_{x}^{0}}+g_{x}^{0}\right), 1\right) \phi_{3}^{0}(x)
$$

depends smoothly on $x=(v, D)$ with 2-regularity and defines a complete maximal surface in $\mathbb{L}^{3} /\left\langle V_{x}\right\rangle$, where

$$
V_{x}=\operatorname{Real}\left[2 \pi i \operatorname{Res}_{0}\left(\Phi(x)^{0}\right)\right]
$$

Since $\phi_{3}^{0}(x)$ is holomorphic at 0 , the vector $V_{x}=\left(w_{x}, 0\right) \in \mathbb{C} \times \mathbb{R}$ is horizontal. Moreover, up to replacing $\phi_{3}^{0}(x)$ by $-\phi_{3}^{0}(x)$ we can suppose that the third coordinate of the flux around the curve $a_{0}(v)$ is positive.

It is straightforward to see that $\theta_{\chi}=\frac{\bar{w}_{x}}{\left|w_{x}\right|}$ and $r_{\chi}=\frac{\varepsilon_{0}}{\left|w_{x}\right|}$ depend smoothly on $\chi$, and so, it follows that $g_{\chi}=\theta_{\chi} g_{x}^{0}$ and $\phi_{3}(\chi)=r_{\chi} \phi_{3}^{0}(x)$ depend smoothly on $\chi$ with 2 and 1-regularity respectively.

To conclude the proof of the claim, observe that

$$
X_{\chi}=q_{0}+\text { Real } \int_{1}\left(\frac{i}{2}\left(\frac{1}{g_{\chi}}-g_{\chi}\right),-\frac{1}{2}\left(\frac{1}{g_{\chi}}+g_{\chi}\right), 1\right) \phi_{3}^{0}(\chi)
$$

depends smoothly on $\chi=\left(x=(v, D), q_{0}, \varepsilon_{0}\right)$ with 2-regularity. Therefore $q_{j}(\chi)=$ $X_{\chi}\left(a_{j}(v)\right)$ and $c(\chi)=g_{\chi}(\infty)$ are smooth functions of $\chi$, and the same holds for $\mathfrak{s}_{2}$. This proves the claim.

By the injectivity of $\mathfrak{s}_{2}$ and the domain invariance theorem, $\mathfrak{s}_{2}\left(\mathfrak{M}_{n}\right)$ is an open domain in $\left.\left(\mathbb{L}^{3} /\langle(1,0,0)\rangle\right)^{n+1} \times\right]-1,+1\left[\right.$. We can then endow $\mathfrak{M}_{n}$ with the unique analytic structure making $\mathfrak{s}_{2}: \mathfrak{M}_{n} \rightarrow \mathfrak{s}_{2}\left(\mathfrak{M}_{n}\right)$ an analytic diffeomorphism. 
To conclude, it remains to check that the action $\mu$ is discontinuous. Indeed, let $\tau: \mathfrak{M}_{n} \rightarrow \mathfrak{M}_{n}$ denote the diffeomorphism given by $\tau(S, \mathfrak{m})=(S, \tau(\mathfrak{m})), \tau \in \mathcal{P}_{n+1}$. Let $\left(S_{0}, \mathfrak{m}_{0}\right) \in \mathfrak{M}_{n}$ and write $\mathfrak{m}_{0}=\left(q_{0}, q_{1}, \ldots, q_{n}\right) \in\left(\mathbb{L}^{3} /\langle(1,0,0)\rangle\right)^{n+1}$. Take a neighborhood $U_{j}$ of $q_{j}$ in $\mathbb{L}^{3} /\langle(1,0,0)\rangle, j=0,1, \ldots, n$, such that $U_{i} \cap U_{j}=\emptyset, i \neq j$, and call $\mathcal{U}=\prod_{j=0}^{n} U_{j}$. Then, it is clear that $\tau\left(\mathfrak{s}_{2}^{-1}(\mathcal{U} \times \mathbb{R})\right) \cap \mathfrak{s}_{2}^{-1}(\mathcal{U} \times \mathbb{R})=\emptyset$, for any $\tau \in \mathcal{P}_{n+1}-\{$ Id $\}$, which proves the discontinuity of $\mu$ and concludes the proof.

To finish, we prove that the underlying topology in $\mathfrak{G}_{n}$ corresponds to the uniform convergence of graphs over compacts subsets of $\left\{x_{3}=0\right\}$.

Theorem 3.3 Let $\left\{G_{k}\right\}_{k \in \mathbb{N}}$ be a sequence in $\mathfrak{G}_{n}$, and $G_{0} \in \mathfrak{G}_{n}$.

Then $\left.\left\{G_{k}\right)\right\}_{k \in \mathbb{N}} \rightarrow G_{0}$ in the topology of $\mathfrak{G}_{n}$ if and only if $\left\{G_{k}\right\}_{k \in \mathbb{N}}$ converges to $G_{0}$ uniformly on compact subsets of $\left\{x_{3}=0\right\}$.

Proof: Suppose $\left\{G_{k}\right\}_{k \in \mathbb{N}} \rightarrow G_{0} \in \mathfrak{G}_{n}$ in the topology of $\mathfrak{G}_{n}$, and choose marks in such a way that $\left\{\left(G_{k}, \mathfrak{m}_{k}\right)\right\}_{k \in \mathbb{N}}$ converges to $\left(G_{0}, \mathfrak{m}_{0}\right)$ in $\mathfrak{M}_{n}$.

Write $\mathcal{E}\left(\left(\mathfrak{G}_{k}, \mathfrak{m}_{k}\right)\right)=\left(x_{k}, q_{0}(k), \epsilon_{k}\right), X_{k}=X_{\left(x_{k}, q_{0}(k), \epsilon_{k}\right)}$ and $x_{k}=\left(v_{k}, D_{k}\right) \in \mathcal{S}_{n}, k \in$ $\mathbb{N} \cup\{0\}$. Observe that, without loss of generality, $\epsilon_{k}=\epsilon_{0}$, for all $k \in \mathbb{N}$.

Since $\left\{\left(x_{k}, q_{0}(k), \epsilon_{k}\right)\right\} \rightarrow\left(x_{0}, q_{0}(0), \epsilon_{0}\right)$ and $X_{\left(x, q_{0}, \epsilon\right)}$ depends smoothly on $\left(x, q_{0}, \epsilon\right)$ with 2-regularity (see the proof of Theorem $[3.2)$, it is not hard to check that $\left\{X_{k}\right\}_{k \in \mathcal{N}}$ diverges uniformly on $k$, that is to say, for any compact $W$ in $\left\{x_{3}=0\right\}$ there is $r>0$ such that $|z+1 / z|>r$ implies $X_{k}(z) \notin W \times \mathbb{R}$, for all $k$.

Let $W$ be any compact domain in the cylinder $\left\{x_{3}=0\right\} \subset \mathbb{L}^{3} /\langle(1,0,0)\rangle$ containing the singularities in $\mathfrak{m}_{0}$ as interior points, and let $W_{k}$ denote the compact set $X_{k}^{-1}(W \times \mathbb{R}) \subset$ $\overline{\Omega\left(v_{k}\right)}-\{0, \infty\}, k \in \mathbb{N} \cup\{0\}$.

As the domains $W_{k}$ are uniformly contained in a compact region of $\mathbb{C}-\{0\}$, then $\left\{W_{k}\right\}_{k \in \mathbb{N}} \rightarrow W_{0}$ in the Hausdorff distance and $X_{k}$ converges uniformly on $W_{0}$ to $X_{0}$. In the last statement we have used that $X_{k}$ can be reflected analytically about the circles in $\partial \Omega\left(v_{k}\right)$, and so all the immersions $X_{k}, k$ large enough, are well defined in a universal neighborhood of $W_{0}$ in $\mathbb{C}$. It is then obvious that the function $u_{k}: \mathbb{R}^{2} \rightarrow \mathbb{R}$ defining the graph $G_{k}$ converges uniformly over $W$ to the function $u_{0}: \mathbb{R}^{2} \rightarrow \mathbb{R}$ defining $G_{0}$ (furthermore, $\left\{v_{k}\right\}_{k \in \mathbb{N}} \rightarrow v_{0}$ implies that $\left\{\mathfrak{m}_{k}\right\}_{k \in \mathbb{N}} \rightarrow \mathfrak{m}_{0}$ ). Since $W$ can be as larger as we want, $\left\{u_{k}\right\}_{k \in \mathbb{N}} \rightarrow u_{0}$ uniformly on compact subsets of $\mathbb{R}^{2}$.

Assume now that the functions $u_{k}:\left\{x_{3}=0\right\} \rightarrow \mathbb{R}$ defining $G_{k}$ converge, as $k \rightarrow \infty$, to the function $u_{0}:\left\{x_{3}=0\right\} \rightarrow \mathbb{R}$ defining $G_{0}$ uniformly on compact subsets of $\left\{x_{3}=0\right\}$.

Let us show that singular points of $G_{0}$ are limits of sequences of singular points of graphs $G_{k}, k \in \mathbb{N}$. Indeed, let $p_{0}=\left(y_{0}, u_{0}\left(y_{0}\right)\right) \in G_{0}$ be a singular point, and without loss of generality, suppose that $p_{0}$ is a downward pointing conelike singularity. By Lemma 2.1. there exists $\epsilon>0$ small enough such that $u_{0}^{-1}\left(\left\{x_{3} \leq u_{0}\left(y_{0}\right)+\epsilon\right\}\right)$ contains a compact component $C_{0}(\epsilon)$ with regular boundary and containing $y_{0}$ as the unique (interior) singular point. Since $\left\{u_{k}\right\}_{k \in \mathbb{N}} \rightarrow u_{0}$ uniformly on compact subsets, $u_{k}^{-1}\left(\left\{x_{3} \leq u_{0}\left(y_{0}\right)+\epsilon\right\}\right)$ must contain a compact component $C_{k}(\epsilon)$ containing $y_{0}$ as well, $k$ large enough. Furthermore, 
$\left\{C_{k}(\epsilon)\right\} \rightarrow C_{0}(\epsilon)$ in the Hausdorff sense, and by the maximum principle $C_{k}(\epsilon)$ must contain at least an interior singular point $y_{k}$ of $u_{k}, k$ large enough. Since $C_{0}(\epsilon)$ converges to $\left\{y_{0}\right\}$ as $\epsilon \rightarrow 0$, we deduce that $\left\{p_{k}:=\left(y_{k}, u_{k}\left(y_{k}\right)\right)\right\}_{k \rightarrow \infty} \rightarrow p_{0}$.

As a consequence, there exist marked graphs $\left(G_{k}, \mathfrak{m}_{k}\right) \in \mathfrak{M}_{n}, k \in \mathbb{N} \cup\{0\}$, such that $\left\{\mathfrak{m}_{k}\right\}_{k \in \mathbb{N}} \rightarrow \mathfrak{m}_{0}$.

Call $c_{k}$ the stereographic projection of the normal vector at $E_{2}$ of $G_{k}, k \in \mathbb{N} \cup\{0\}$, and let us see that $\left\{c_{k}\right\} \rightarrow c_{0}$. Indeed, take $C<0$ small enough such that $\left\{x_{3}=0, x_{2} \leq C\right\}$ does not contain any singular point of $u_{k}, k \in \mathbb{N} \cup\{0\}$, let $\gamma$ be a circle in $\left\{x_{3}=0, x_{2} \leq C\right\}$ and let $A$ denote a closed tubular neighborhood of $\gamma$ in $\left\{x_{3}=0\right\}$ not containing any singular point of $u_{0}$. It is well known that $u_{k}-u_{0}$ is solution of a uniformly elliptic linear equation $L_{k}\left(u_{k}-u_{0}\right)=0$ over $A, k$ large enough. Moreover, the fact that the functions $\frac{1}{1-\left|\nabla u_{k}\right|}$, $k \in \mathbb{N}$, are uniformly bounded on $A$ (see [1]) guarantee that the coefficients of operators $L_{k}, k \in \mathbb{N}$, are uniformly bounded too. Therefore, since $\left\{u_{k}\right\}_{k \in \mathbb{N}} \rightarrow u_{0}$ uniformly on $A$, the classical Schauder estimates (9] p. 93) imply that $\left\{u_{k}\right\}_{k \in \mathbb{N}} \rightarrow u_{0}$ in the $C^{2}$ norm on $A$. In particular,

$$
\left\{n_{k}:=\int_{\gamma} \nu_{k}\left(s_{k}\right) d s_{k}\right\}_{k \in \mathbb{N}} \rightarrow n_{0}:=\int_{\gamma} \nu_{0}\left(s_{0}\right) d s_{0},
$$

where $\nu_{k}$ and $s_{k}$ are the conormal vector and the arc-length parameter along $\gamma$ in $G_{k}$, respectively, for any $k \in \mathbb{N} \cup\{0\}$. Since the normal vector at $E_{2}$ of $G_{k}$ lies in $\left\{x_{1}=0\right\}$ and is orthogonal to $n_{k}, k \in \mathbb{N} \cup\{0\}$, we infer that $\left\{c_{k}\right\} \rightarrow c_{0}$.

Since $\mathfrak{s}_{2}: \mathfrak{M}_{n} \rightarrow \mathfrak{s}_{2}\left(\mathfrak{M}_{n}\right) \subset \mathbb{R}^{3 n+4}$ is an homeomorphism, $\left\{\left(G_{k}, \mathfrak{m}_{k}\right)\right\}_{k \in \mathbb{N}} \rightarrow\left(G_{0}, \mathfrak{m}_{0}\right)$ in the manifold $\mathfrak{M}_{n}$, and so, $\left\{G_{k}\right\}_{k \in \mathbb{N}} \rightarrow G_{0}$ in the manifold $\mathfrak{G}_{n}$. This concludes the proof.

\section{References}

[1] R. Bartnik and L. Simon: Spacelike hypersurfaceswith prescribed boundary values and mean curvature. Comm. Math. Phys.,Vol. 87(1982/83), 131-152.

[2] E. Calabi.: Examples of the Bernstein problem for some nonlinear equations. Proc. Symp. Pure Math., Vol. 15, (1970), 223-230.

[3] S. Y. Cheng and S. T. Yau.: Maximal space-like hypersurfaces in the LorentzMinkowski spaces. Ann. of Math. (2), Vol. 104 (1976), 407-419.

[4] K. Ecker.: Area maximizing hypersurfaces in Minkowski space having an isolated singularity. Manuscripta Math., Vol. 56 (1986), 375-397.

[5] F. J. M. Estudillo and A. Romero.: Generalized maximal surfaces in the LorentzMinkowski space $\mathbb{L}^{3}$ Math. Proc. Camb. Phil. Soc. 111, (1992), 515-524.

[6] H. M. Farkas, I. Kra.: Riemann surfaces. Graduate Texts in Math., 72, Springer Verlag, Berlin, 1980. 
[7] I. Fernández and F. J. López.: Periodic Maximal surfaces in the Lorentz-Minkowski space $\mathbb{L}^{3}$. Preprint.

[8] I. Fernández, F. J. López and R. Souam.: The space of complete embedded maximal surfaces with isolated singularities in the 3-dimensional Lorentz-Minkowski space $\mathbb{L}^{3}$. Preprint. Link: http://arxiv.org/PS_cache/math/pdf/0311/0311330.pdf

[9] D. Gilbarg and N. S. Trudinger.: Elliptic partial differential equations of second order. Springer-Verlag, (1977).

[10] A. A. Klyachin.: Description of a set of entire solutions with singularities of the equation of maximal surfaces. (Russian) Mat. Sb. 194 (2003), no. 7, 83-104; translation in Sb. Math. 194 (2003), no. 7-8, 1035-1054.

[11] V. A. Klyachin and V.M. Miklyukov.: Geometric structures of tubes and bands of zero mean curvature in Minkowski space. Annales Academia Scientiarum Fennicae Mathematica, 28 (2003) 239-270

[12] O. Kobayashi.: Maximal surfaces with conelike singularities. J. Math. Soc. Japan 36 (1984), no. 4, 609-617

[13] F. J. López, R. López and R. Souam.: Maximal surfaces of Riemann type in LorentzMinkowski space $\mathbb{L}^{3}$. Michigan J. of Math., Vol. 47 (2000), 469-497.

[14] M. Umehara and K. Yamada: Maximal surfaces with singularities in Minkowski space. Preprint.

[15] J. Wolf: Spaces of Constant curvature. McGraw-Hill, New York (1967).

[16] B. O'Neill: Semmi-riemannian geometry. Academic Press (1983).

ISABEL FERNANDEZ, FRANCISCO J. LOPEZ,

Departamento de Geometría y Topología

Facultad de Ciencias, Universidad de Granada

18071 - GRANADA (SPAIN)

e-mail:(first author) isafer@ugr.es, (second author) fjlopez@ugr.es

RABAH SOUAM,

Institut de Mathématiques de Jussieu-CNRS UMR 7586

Université Paris 7

Case 7012

2,place Jussieu

75251 Paris Cedex 05, France

e-mail: souam@math.jussieu.fr 